ARTICLE

DOI: $10.1038 /$ s41467-018-07329-0

\title{
Invasive Salmonella exploits divergent immune evasion strategies in infected and bystander dendritic cell subsets
}

Anna Aulicino ${ }^{1,2}$, Kevin C. Rue-Albrecht (10 ${ }^{3}$, Lorena Preciado-Llanes ${ }^{1,2}$, Giorgio Napolitani ${ }^{1}$, Neil Ashley ${ }^{4}$, Adam Cribbs ${ }^{5}$, Jana Koth ${ }^{6}$, B. Christoffer Lagerholm ${ }^{6}$, Tim Ambrose ${ }^{1,2}$, Melita A. Gordon ${ }^{7,8}$, David Sims ${ }^{5}$ \& Alison Simmons (1D) 1,2

Non-typhoidal Salmonella (NTS) are highly prevalent food-borne pathogens. Recently, a highly invasive, multi-drug resistant S. Typhimurium, ST313, emerged as a major cause of bacteraemia in children and immunosuppressed adults, however the pathogenic mechanisms remain unclear. Here, we utilize invasive and non-invasive Salmonella strains combined with single-cell RNA-sequencing to study the transcriptome of individual infected and bystander monocyte-derived dendritic cells (MoDCs) implicated in disseminating invasive ST313. Compared with non-invasive Salmonella, ST313 directs a highly heterogeneous innate immune response. Bystander MoDCs exhibit a hyper-activated profile potentially diverting adaptive immunity away from infected cells. MoDCs harbouring invasive Salmonella display higher expression of IL10 and MARCH1 concomitant with lower expression of CD83 to evade adaptive immune detection. Finally, we demonstrate how these mechanisms conjointly restrain MoDC-mediated activation of Salmonella-specific CD4 ${ }^{+} \mathrm{T}$ cell clones. Here, we show how invasive ST313 exploits discrete evasion strategies within infected and bystander MoDCs to mediate its dissemination in vivo.

\footnotetext{
${ }^{1}$ MRC Human Immunology Unit, MRC Weatherall Institute of Molecular Medicine, University of Oxford, Oxford OX3 9DS, UK. ${ }^{2}$ Translational Gastroenterology Unit, John Radcliffe Hospital, Headington, Oxford OX3 9DU, UK. ${ }^{3}$ Kennedy Institute of Rheumatology, Nuffield Department of Orthopaedics, Rheumatology and Musculoskeletal Sciences, University of Oxford, Headington, Oxford OX3 7FY, UK. ${ }^{4}$ MRC Molecular Haematology Unit, MRC Weatherall Institute of Molecular Medicine, University of Oxford and BRC Blood Theme, NIHR Oxford Biomedical Centre, Oxford OX3 9DS, UK. ${ }^{5}$ MRC WIMM Centre for Computational Biology, MRC Weatherall Institute of Molecular medicine, University of Oxford, Oxford OX3 9DS, UK. ${ }^{6}$ MRC Human Immunology Unit and Wolfson Imaging Centre, MRC Weatherall Institute of Molecular Medicine, University of Oxford, Oxford OX3 9DS, UK. ${ }^{7}$ Institute of Infection and Global Health, University of Liverpool, 8 W Derby St, Liverpool L7 3EA, UK. ${ }^{8}$ Malawi-Liverpool Wellcome Trust Clinical Research Programme, Blantyre, Malawi. These authors contributed equally: Anna Aulicino, Kevin C. Rue-Albrecht Correspondence and requests for materials should be addressed to A.S. (email: alison.simmons@ndm.ox.ac.uk)
} 
$\mathrm{N}$ on-typhoidal Salmonella (NTS) are among the most common food-borne pathogens, which cause 93 million cases of gastroenteritis each year, including 155,000 deaths ${ }^{1,2}$. The most frequently reported, Salmonella enterica serovar Typhimurium (S. Typhimurium), causes localized, selflimiting gastroenteritis and infects both humans and animals ${ }^{3}$. Recently, a highly invasive multi-drug resistant $S$. Typhimurium of a distinct multi-locus sequence type (MLST), ST313, has emerged. Its prevalence across sub-Saharan Africa became a major cause of lethal bacteraemia in young malnourished anaemic children and individuals affected by concomitant diseases such as malaria or $\mathrm{HIV}^{4,5}$. S. Typhimurium ST313 pathovar D23580 (STM-D23580) is a representative bloodstream clinical isolate, which exhibits genomic degradation resembling the human-restricted pathogen S. Typhi ${ }^{4}$. How ST313 usurps mucosal immune defences to achieve an invasive phenotype in susceptible individuals is unclear. In particular, whether it usurps dendritic cell (DC) functions to avoid adaptive immunity has not been elucidated.

DCs are key professional antigen-presenting cells (APCs) of the immune system. Activating pattern recognition receptors (PRRs) expressed in DCs stimulates their maturation and induces antigen-specific immunity ${ }^{6,7}$. The primed immune response critically depends on the specific signalling context triggered by invading bacteria. Thus, the ability of pathogenic bacteria to subvert DC functions and prevent $\mathrm{T}$ cell recognition contributes to their survival and dissemination within susceptible hosts and cell subsets. Salmonella can modulate DC functions ${ }^{8-10}$. However, it remains unclear whether individual DCs differentially recognize genetically similar $S$. Typhimurium strains and how differential innate immune sensing produces distinct functional outcomes. Furthermore, the mechanisms of action that facilitate ST313 evasion strategies in specific DCs in human disease remain unclear.

Transcriptomics approaches are utilized to survey and identify host cellular signalling pathways in response to intracellular bacteria ${ }^{11}$. Earlier analyses of bulk samples amalgamated the gene expression profiles of millions of cells and did not account for the phenotypic diversity between individual cells. In contrast, single-cell RNA-sequencing (scRNA-seq) has emerged as a powerful tool to profile previously inaccessible cell-to-cell transcriptional variability on a genome-wide scale ${ }^{12-14}$. Pioneering scRNA-seq analyses of lipopolysaccharide (LPS)-stimulated murine bone-marrowderived DCs revealed an unexpected degree of bimodal expression among immune genes exposed to uniform pathogen-associated molecular patterns (PAMPs), otherwise detected at seemingly consistent high levels as average population measurements ${ }^{15}$. More recently, scRNA-seq revealed considerable heterogeneity in responses of individual murine macrophages to variations in intracellular Salmonella growth ${ }^{16}$.

Here, we combine fluorescent-activated cell sorting (FACS) and scRNA-seq to survey the transcriptome of individual human MoDCs challenged with invasive or non-invasive Salmonella. Using this system, we measure the transcriptional profile with high sensitivity and full-length gene coverage. We distinguish infected MoDCs, in which Salmonella persists and adapts to the host, from neighbouring cells, either stimulated by bacterial PAMPs or that have engulfed and processed bacterial moieties. We elucidate the mechanisms of action that ST313 utilizes to disseminate in specific MoDC subsets. Together, our scRNA-seq results reveal the mechanisms of cell-intrinsic host adaption exploited by Salmonella ST313. These mechanisms, in conjunction with bystander hyper-activation, provide insight for its invasive behaviour in immunocompromised hosts.

\section{Results}

Single-cell RNA-sequencing of challenged human MoDCs. To profile the transcriptional response of individual human MoDCs infected with bacteria and compare it with that of bystander cells, we labelled STM-LT2 and STM-D23580 with CellTrace ${ }^{\text {TM }}$ Violet Cell Proliferation dye prior to infection (Fig. 1a and Supplementary Figure 1). MoDCs that engulfed Salmonella could be identified by their emitted Violet fluorescence, while bystander MoDCs exhibited no Violet signal (Supplementary Figure 2). Internalization of both bacterial strains was also confirmed by confocal microscopy using a specific anti-Salmonella antibody (Supplementary Figure 3).

We confirmed the presence of live Salmonella within infected cells by sorting MoDCs by their fluorescence phenotype and enumerating intracellular bacteria after cell lysis. Infected cells showed constant numbers of intracellular bacteria over time, while no or very few viable bacteria were recovered from bystander MoDCs (Supplementary Figure 4). STM-LT2 and STM-D23580 demonstrated equivalent abilities to survive and multiply within MoDCs, and no significant differences were observed in the number of CFU between bacterial strains at each time point (Supplementary Figure 5A). The percentage of uptake and survival was also comparable for both strains (Supplementary Figure 5B and 5C). Moreover, no significant differences were observed in the viability of MoDCs infected with the two bacterial strains during the course of the infection (Supplementary Figure 5D).

Individual infected or bystander MoDCs and uninfected MoDCs from mock-treated cultures were isolated by FACS sorting at 2, 4 and $6 \mathrm{~h}$ after infection. We then performed scRNAseq on single sorted MoDCs according to the Smart-seq2 protocol $^{17}$ (Fig. 1a). In total, we profiled the transcriptome of 373 human MoDCs across 15 conditions (23-31 cells per condition; Supplementary Data 1$)$. After removing 31 cells $(\sim 8$ $\%)$ through stringent quality metrics (Supplementary Figure 6), 342 cells remained for downstream analyses (18-30 cells per condition, Supplementary Tables 1 and 2). Notably, we observed similar distributions of average $\log _{10}$-transformed read count per million (CPM) across all conditions. We detected an average of 10,820 genes (range: 9698-12,143) above an average 1 CPM in at least one experimental group and an average of 4221 genes (range: 3636-4827) below the $1 \mathrm{CPM}$ average, respectively (Supplementary Figure 7A).

Transcriptional reprogramming following Salmonella infection. We applied the diffusion map non-linear dimensionality reduction method to reduce the high-dimensional normalized expression data set and to visualize relations between data points in a low-dimensional $\operatorname{plot}^{18}$. The resulting embedding highlights the progression of cells challenged with bacteria through markedly distinct stages, reflecting the sequential time points of the experiment. Notably, mock-infected cells displayed a shorter and continuous trajectory illustrating a more limited transcriptional drift in the absence of bacterial stimuli (Fig. 1b).

To identify transcriptomics changes taking place in MoDCs over the course of infection, we ordered all 342 cells in pseudotime using a set of 2,759 genes differentially expressed between Salmonella-challenged cells at $2 \mathrm{~h}$ and $6 \mathrm{~h}$ p.i. ( $q$-value $<$ 0.001 ) and identified genes that changed as a function of pseudotime across all cells (3188 genes, $q$-value $<0.001$; Fig. 1c and Supplementary Data 2).

Genes partitioned into three broad co-regulated modules based on their single-cell expression profiles. Module 1 was strongly enriched for Gene Ontology (GO) terms related to the host immune response (Innate Immune Response, Cytokine-mediated 
signalling pathway; Bonferroni-corrected $P$-value $<1 \mathrm{e}-39)$ and included genes known to be activated upon bacterial stimulation and internalization, such as pro-inflammatory cytokines (IL12B, $I L 6, I L 1 B)$ and the Lysosomal Associated Membrane Protein 3 (LAMP3) (Fig. 1c and Supplementary Data 3). In contrast, Module 2 and Module 3, which contained genes downregulated in challenged MoDCs over the course of the infection, were enriched for GO terms associated with metabolic activity (Lipid Metablic Process, Oxidation-Reduction Process; Bonferronicorrected $P$-value $<1 \mathrm{e}-03$ ), and protein synthesis (Ribosomal Biogenesis, Translational Initiation; Bonferroni-corrected $P$-value $<1 \mathrm{e}-06$ ), respectively (Fig. $1 \mathrm{c}$ and Supplementary Data 3 ). These findings are consistent with previous studies showing that professional phagocytes respond to bacterial stimuli by reorganizing their metabolic activities ${ }^{19}$, in addition to mounting immune responses. Also, inhibition of host protein synthesis is a common strategy used by many viral and bacterial pathogens to disrupt host gene expression ${ }^{20,21}$.

Increasing MoDC heterogeneity over the course of infection. As the marked temporal evolution of the transcriptional profile of Salmonella-challenged MoDCs masked the expectedly subtler differences expected in gene expression between bacterial strains (i.e., STM-LT2 vs. STM-D23580) and infection status (i.e., infected vs. bystander), we examined data collected at each time point in separate downstream analyses. To identify major cellular phenotypes, we identified unsupervised clusters at each time point by rank correlation between single-cell gene expression profiles using the scran package ${ }^{22}$ (Supplementary Table 3).

At $2 \mathrm{~h}$ p.i. (Fig. 2), cluster 1 contained a balanced proportion of mock-uninfected and challenged MoDCs; cluster 3 was largely dominated by mock-uninfected cells and cluster 2 uniquely contained Salmonella-challenged MoDCs, possibly representing a small proportion of precocious activated MoDCs ${ }^{23}$ (Supplementary Table 3). In agreement with this hypothesis, differential expression analysis (using the scde package ${ }^{24}$ ) revealed significant enrichment of genes involved in Lipid Antigen Presentation (Bonferroni-corrected $P$-value 5.94e-03; CD1A, CD1B, CD1C) as well as increased expression of surface markers (CD83 and CD40) and cytokines (TNF), which are known markers of MoDC maturation, compared to all other cells at that time point (Fig. 2b and Supplementary Data 4 and 5).

At $4 \mathrm{~h}$ p.i. the different transcriptional profiles of uninfected, infected and bystander cells were more evident (Fig. 2). Cluster 3 contained all uninfected cells, with the exception of a single STMD23580 bystander cell. Cluster 1 consisted of $74.5 \%$ of infected cells and cluster 2 was formed by $75.5 \%$ of bystander cells (Supplementary Table 3). Remarkably, at this stage of the infection, the comparison of unsupervised clusters 1 and 2 replicated a significant proportion of DE genes identified from the direct comparison of infected and bystander MoDCs in both STM-LT2 and STM-D23580 infections (Supplementary Figure 8A and Supplementary Data 4 and 6). Such findings indicate that clear distinctive transcriptional reprogramming events take place in cells that engulfed bacteria, relative to cells that were only exposed to extracellular cues. Conversely, the non-negligible proportion of cells from each experimental group split over multiple unsupervised clusters indicated a certain level of variability between cells exposed to uniform experimental stimuli.

Analysis of cluster 1 marker genes revealed a predominance of GO terms related to Immune Response and Intracellular Stress (Bonferroni-corrected $P$-value $<1 \mathrm{e}-06$ ). In particular, this cluster was enriched for the lysosomal proteases CTSB, CTSL, CTSS suggesting an increased proteolytic activity that may occur in phagocytic cells harbouring bacteria. Cluster 2, containing most of the bystander cells, was instead enriched in LPS-induced genes (Bonferroni-corrected $P$-value $<1.19 \mathrm{e}-11$ ), compatible with extracellular stimuli. Cells in this cluster also express typical dendritic cells maturation markers (CD40, CD83) and increased levels of TNF and its targets (i.e. TNFAIP2, TNFAIP3, TNFAIP8) (Supplementary Data 4 and 5).

At 6 h p.i., cluster 3 was exclusively constituted by uninfected cells. Cluster 1 and cluster 2 revealed a more heterogeneous composition than that observed at $4 \mathrm{~h}$ p.i., emphasizing the complexity of cell-to cell variability (Fig. 2 and Supplementary Table 3). Genes induced in MoDCs of cluster 1 were enriched for Antiviral Defence factors, including interferon targets (Bonferroni-corrected $P$-value $<4.72 \mathrm{e}-11)$, whereas cells of cluster 2 were enriched for inflammatory genes and NF-kB targets (Bonferroni-corrected $P$-value $<4.14 \mathrm{e}-20) \quad$ (Supplementary Data 5).

Interestingly, cluster 2 included most of the STM-D23580 bystander MoDCs (77.7\%), in contrast to a minority of MoDCs infected with or exposed to STM-LT2 (31.8\%), thus emphasizing larger differences between infected and bystander MoDCs challenged with STM-D23580 than those observed with STMLT2 (Supplementary Table 3 and Supplementary Figure 8B). Consistent with this observation, we found $151 \mathrm{DE}$ genes between infected and bystander MoDCs challenged with STM-D23580, compared to only $85 \mathrm{DE}$ genes identified between infected and bystander MoDCs challenged with STM-LT2 at $6 \mathrm{~h}$ p.i. (Supplementary Figure 8C). Taken together, these results highlight more pronounced differences between invasive Salmonella infected cells and their equivalent bystander cells.

STM-D23580 amplifies infected and bystander MoDC differences. Next, we compared the differences between infected and bystander cells challenged with either Salmonella strain, to characterize the transcriptional reprogramming events common or specific to cells harbouring each of the two bacterial strains, in relation to their respective bystander cells.

ScRNA-seq revealed strain-specific changes and differential pathway activities in infected and bystander MoDCs. While certain genes were commonly up- or downregulated in infected and bystander cells by both bacterial strains, several genes were uniquely DE between infected and bystander cells only by STMD23580 or STM-LT2. Indeed, these data suggest that the invasive strain achieves successful host infection by affecting common as well as disparate host pathways (Fig. 3 and Supplementary Data 7).

For instance, at $2 \mathrm{~h}$ p.i., STM-D23580-infected cells specifically upregulated the Membrane-Associated Ring-CH-Type Finger 8 MARCH8, which has been recently shown to be a target of the Salmonella effector protein $\mathrm{SteD}^{25}$, as well as RAB29, a GTPase recruited to the $S$. Typhi-containing vacuole but not to vacuoles containing broad-host Salmonella ${ }^{26}$

Interestingly, at the later stages of our infection model, genes essential for correct antigen presentation (CD83, CD40, HLA$D O A$ ) were downregulated exclusively in STM-D23580-infected cells relative to bystander cells.

STM-D23580 enhances an inflammatory state in bystander MoDCs. At $6 \mathrm{~h}$ p.i, our unsupervised clustering approach revealed an enrichment for STM-D23580 bystander MoDCs in cluster $2(77.7 \%)$ compared to a more balanced representation of other cells across clusters 1 and 2 (Fig. 2 and Supplementary Table 3 ). These results suggest that most cells exposed to PAMPs from the invasive STM-D23580 engage in a distinct transcriptional program from that of STM-LT2 bystander MoDCs. As compared to STM-LT2, STM-D23580 bystander cells revealed a 
a

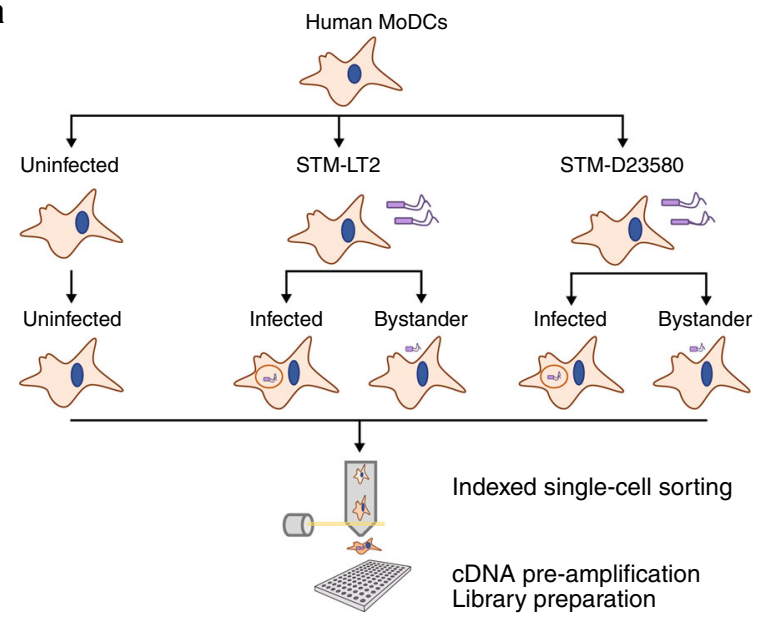

Deep single-cell RNA-sequencing (SMART-seq2) $373 \mathrm{MoDCs}, \sim 1.5 \mathrm{M}$ reads/cell, $\sim 5028$ genes/cell

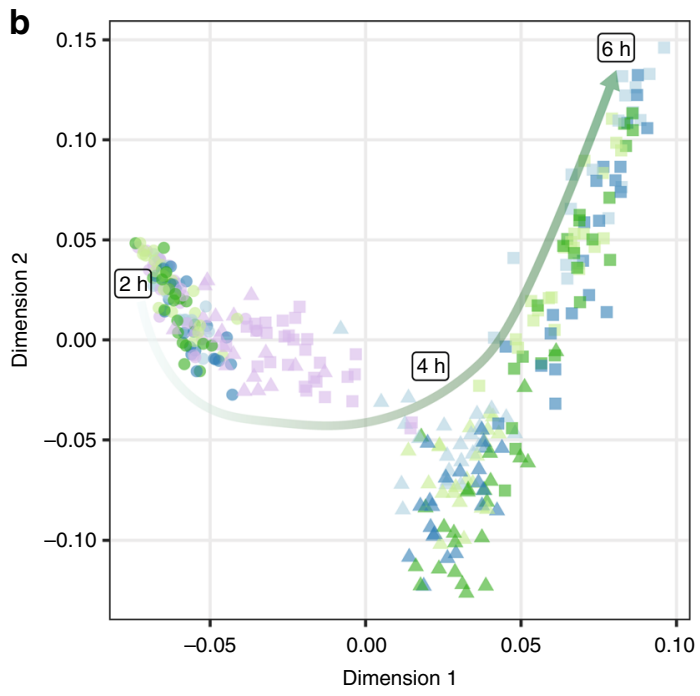

$\begin{array}{ll}\text { Mock uninfected } & \text { STM-LT2 bystander } \\ \text { STM-D23580 Bystander }\end{array}$

$2 \mathrm{~h} \Delta 4 \mathrm{~h} \square 6 \mathrm{~h}$

C
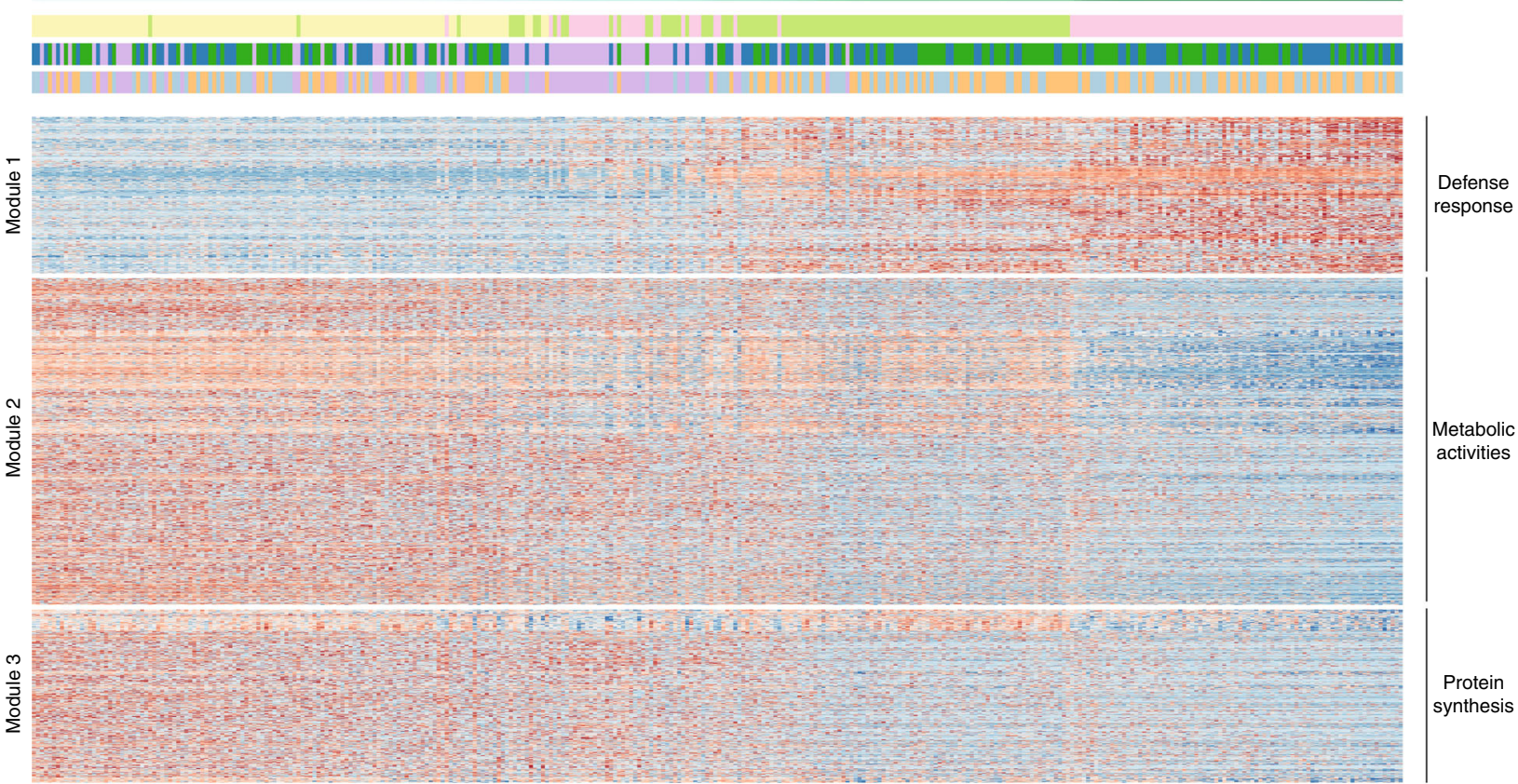

\begin{tabular}{|c|c|c|c|c|c|}
\hline & unt + 1) & Pseudotime & Time & Infection & Status \\
\hline I & 1 & 1 & $\begin{array}{l}2 \mathrm{~h} \\
4 \mathrm{~h}\end{array}$ & $\begin{array}{l}\text { Mock } \\
\text { STM-LT2 }\end{array}$ & $\begin{array}{l}\text { Uninfected } \\
\text { Bystander }\end{array}$ \\
\hline & $10 \quad 15$ & 010203040 & $6 \mathrm{~h}$ & STM-D23580 & Infected \\
\hline
\end{tabular}

Fig. 1 Single-cell transcriptomics analysis of human MoDCs challenged with invasive or non-invasive Salmonella. a Schematic representation of the experimental design. Human MoDCs were challenged with labelled STM-LT2 or STM-D23580 bacterial strains. At 2, 4 and $6 \mathrm{~h}$ post infection (p.i.), infected and bystander cells were sorted and processed according to the Smart-seq2 protocol. $\mathbf{b}$ The diffusion map non-linear dimensionality reduction technique was applied on the first 50 components of a principal component analysis (PCA) computed on the 500 most variable genes to visualize the high-dimensional relations between cells in the data set. The diffusion map reveals distinct cell populations that progress through the three time points of the experimental design. c Heat map displaying $\log _{2}$-transformed normalized counts for 3188 genes that significantly change with pseudotime across all single-cells ( $q$-value $<0.001)$. Pseudotime was computed from a set of 2759 genes differentially expressed between Salmonella-challenged cells at 2 and $6 \mathrm{~h}$ p.i. ( $q$-value $<0.001)$. Experimental metadata (i.e., time point, infection, status) are indicated for each cell as colour bars above the heat map 
a

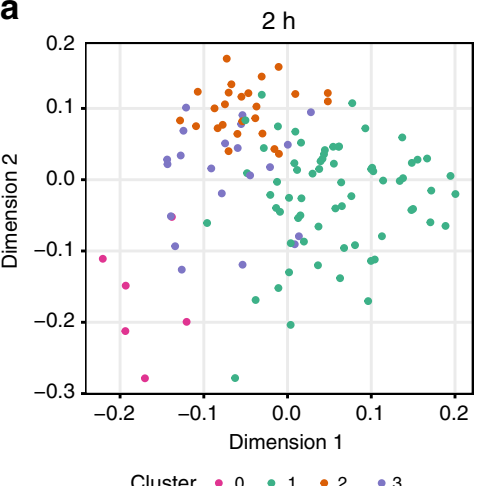

\section{b}

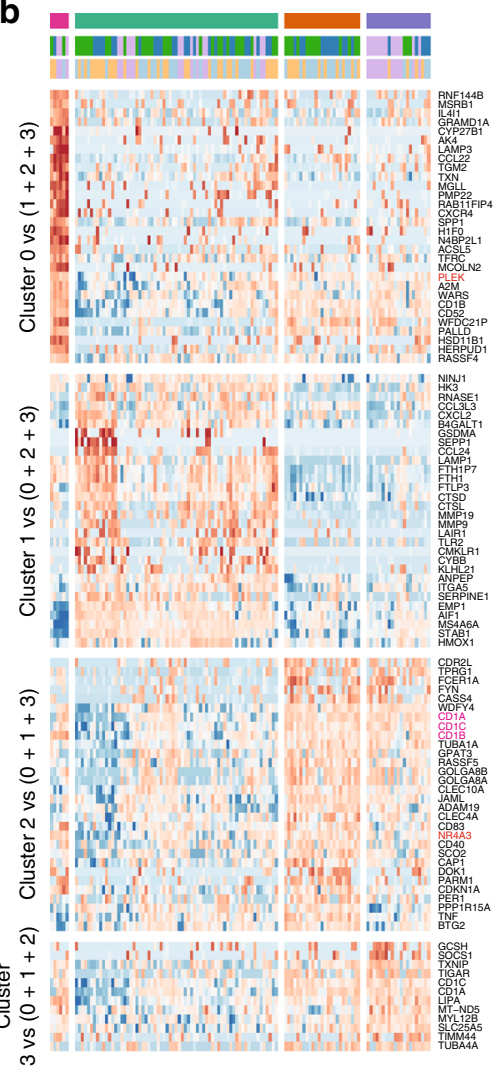

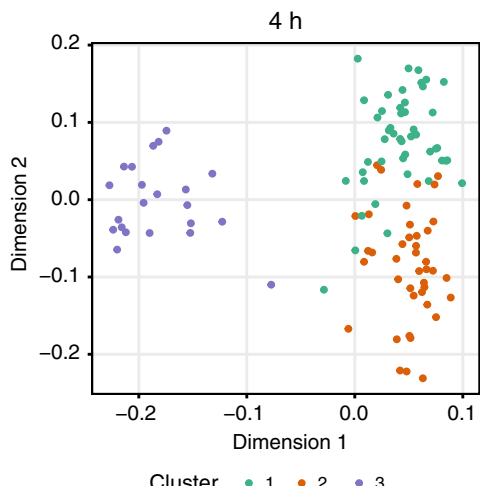

Cluster $1 \cdot 2 \cdot 3$

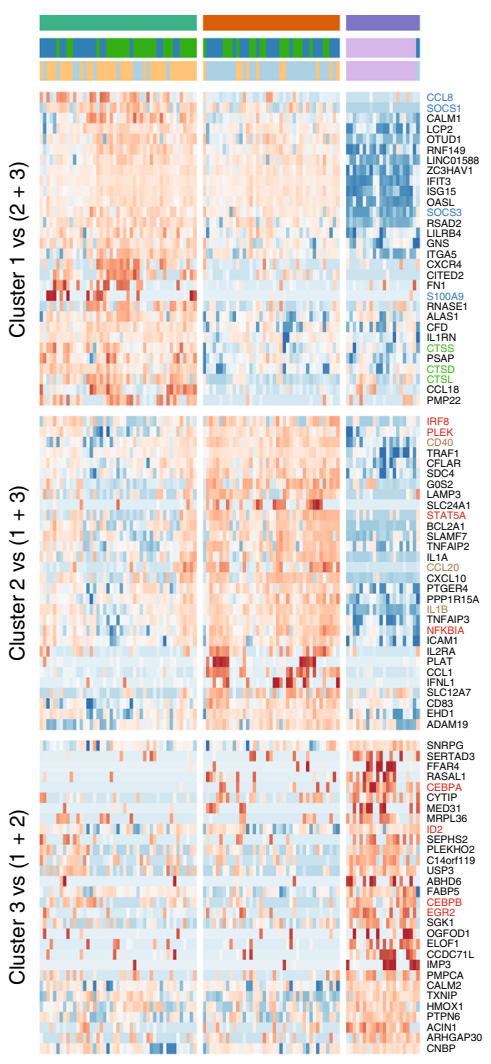

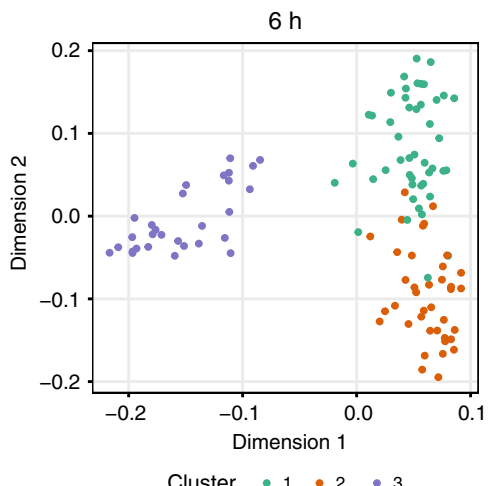

Cluster $\bullet 1 \cdot 2 \cdot 3$

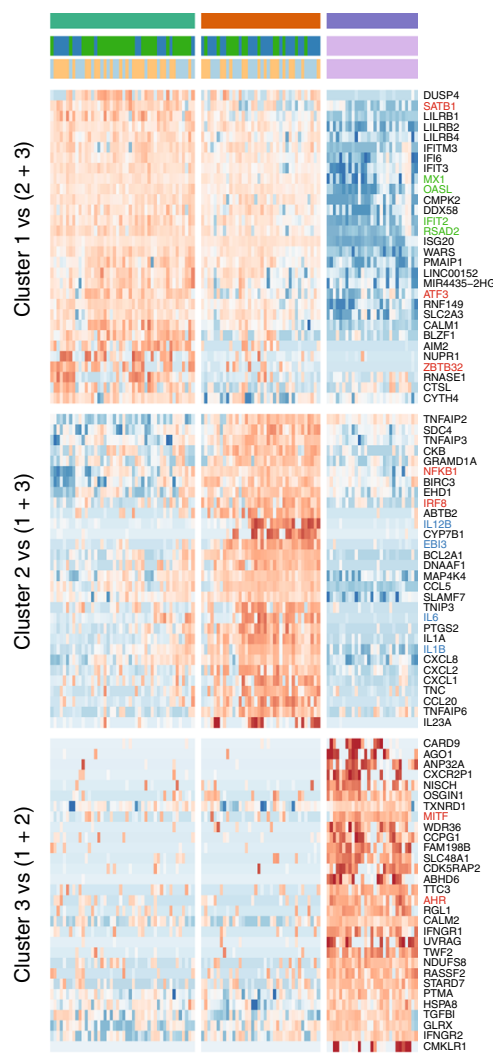

Status

Uninfected

Bystander

Infected

STM-D23580

Gene ontology terms

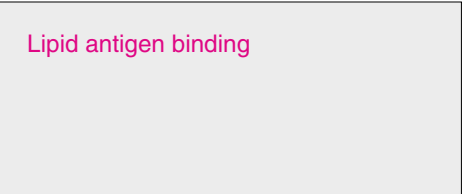

Defense response to virus

Cytokine-mediated signalling pathway

Fig. 2 Unsupervised clustering reveals variability within infected and bystander cells. a Diffusion maps highlighting the unsupervised clusters identified by rank correlations between gene expression profiles of cells at each time point separately. Diffusion maps were computed on the first 50 components of a principal component analysis (PCA) using the 500 most variable genes identified within cells at each time point separately. $\mathbf{b}$ Heat maps displaying up to 30 significant marker genes identified as DE genes $(P$-value $<0.01)$ between each unsupervised cluster and all other cells at the corresponding time point ( 2,4 and $6 \mathrm{~h} \mathrm{p.i,} \mathrm{respectively).} \mathrm{Rows} \mathrm{and} \mathrm{columns} \mathrm{were} \mathrm{clustered} \mathrm{using} \mathrm{Euclidian} \mathrm{distance} \mathrm{and} \mathrm{complete} \mathrm{linkage} \mathrm{method} \mathrm{applied} \mathrm{to} \mathrm{the} \mathrm{row-scaled} \mathrm{values}$ across all heat map panels at that time point. Enriched GO categories and associated genes are indicated by colors. Transcription factors are highlighted in red 

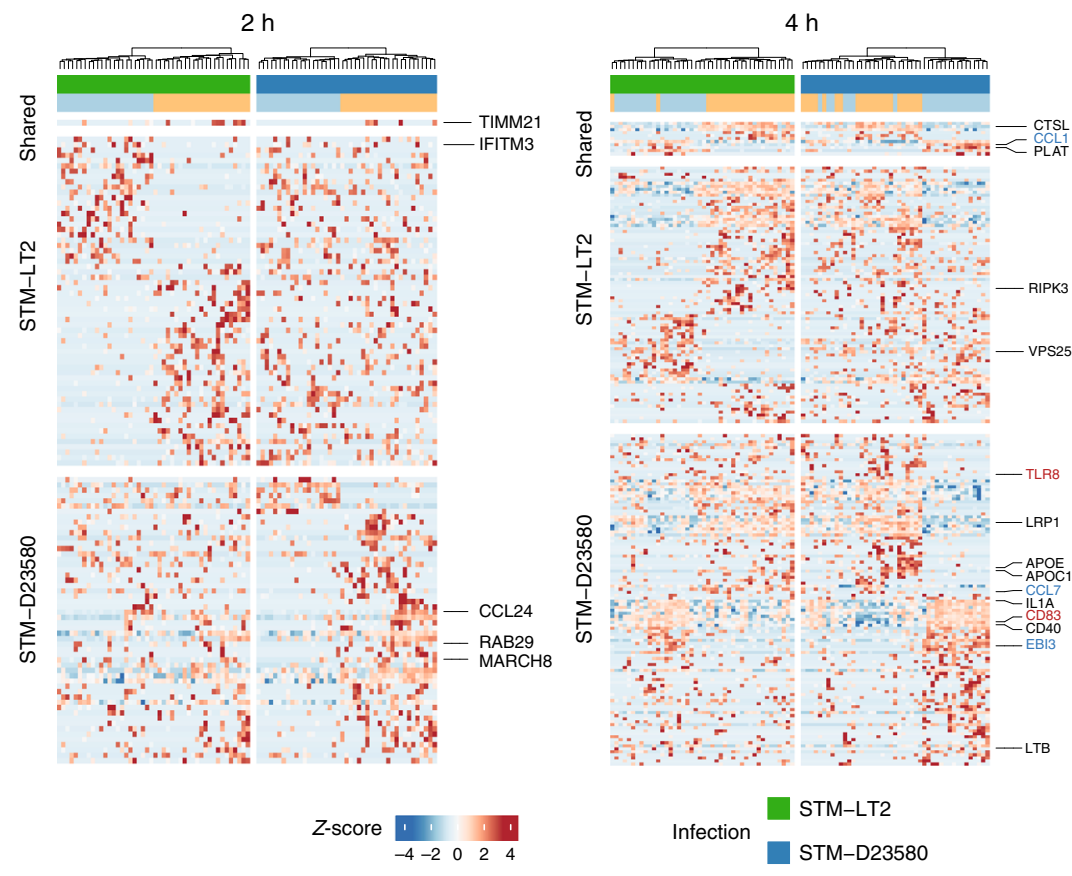

Gene ontology terms

Regulation of multicellular
organismal process
Cytokine receptor binding
External side of plasma membrane
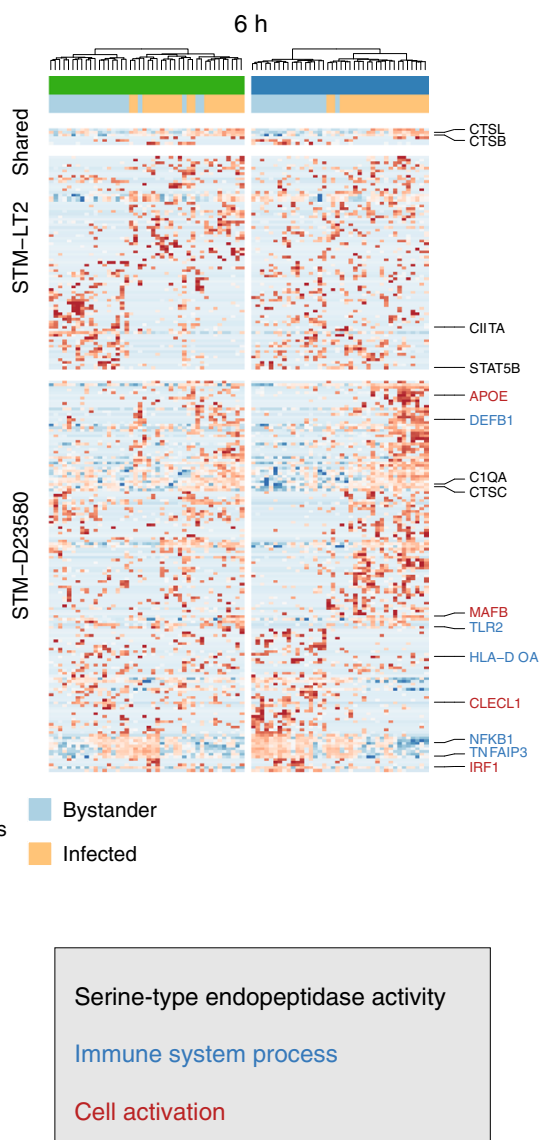

Fig. 3 STM-D2350 and STM-LT2 induce divergent single-cell profiles in infected and bystander cells. Heat maps displaying significant DE genes ( $P$-value < 0.01 ) identified between infected and bystander cells at time points 2,4 and $6 \mathrm{~h} \mathrm{p.i,} \mathrm{respectively.} \mathrm{Rows} \mathrm{and} \mathrm{columns} \mathrm{were} \mathrm{clustered} \mathrm{using} \mathrm{Euclidian}$ distance and Ward's clustering criterion applied to the row-scaled log-transformed normalized counts across all heat map panels at that time point. Enriched GO categories and associated genes are indicated by colors

significantly higher expression of the typical pro-inflammatory cytokines (IL1B, IL12B, CXCL8) (Fig. 4a and Supplementary Data 6 and 8). Additionally, the transcription factors TNFAIP3, TNIP3, BIRC3, which activate inflammatory signalling cascades, were upregulated in STM-D23580 bystander cells as compared to STM-LT2 (Fig. 4b and Supplementary Data 6 and 8). Exposure to invasive Salmonella also activated the transcription of genes known to inhibit apoptotic processes (BCL2L1) and regulate NF$\mathrm{kB}$ signalling $(I K B K B)$. Conversely, STM-D23580 bystander MoDCs expressed ATF3, a key negative regulator of cytokine expression in immune cells ${ }^{27,28}$, at levels no different than uninfected cells. Such observation contrasts with a significant ATF3 upregulation among STM-LT2 bystander cells. Together, these results demonstrate an impaired transcriptional regulation in cells exposed to the invasive strain. We next explored how STM-D23580 might direct enhanced pro-inflammatory response in exposed MoDCs. We assessed whether surface PAMPs might play a role by exposing MoDCs to heat-killed (HK) Salmonella treated with DNAse, RNAse and Proteinase K, and assayed proinflammatory cytokine responses. Higher IL-1 $\beta$ and IL-12p40 secretion was detected in MoDCs stimulated with HK STMD23580 as compared to HK STM-LT2 (Supplementary Figure 9) suggesting that STM-D23580 surface moieties may play some part in this effect.

Differences between STM-D23580- and STM-LT2-infected MoDCs. To identify unique differences between MoDCs infected with either bacterial strain, we directly compared STM-
D23580- and STM-LT2-infected cells at each time point (Fig. 5a and Supplementary Data 6). During the early and intermediate stages of the infection ( 2 and $4 \mathrm{~h}$ p.i.), we found that STMD23580-infected cells displayed increased expression of the apoptosis-related cysteine peptidase Caspase 3 (CASP3), the vacuolar protein sorting 25 (VPS25) required for multivesicular body (MVB) formation, and a group of genes involved in the metabolism of fatty acids (MUT, CHST13, APOBEC3A). Conversely, genes that facilitate ubiquitination and proteasomal degradation (KLHL2, TRIM13) were downregulated in STM-D23580 as compared with STM-LT2-infected cells. Consistent with progressive bacterial adaptation to the host intracellular environment, we found increased gene expression changes later in infection (6h p.i.). Interestingly, the RING Type E3-ubiquitin Transferases $M A R C H 1$ and $R N F 139$, required for ubiquitination of MHC-II and MHC-I molecules ${ }^{29,30}$ respectively, were upregulated in STM-D23580 as compared to STM-LT2-infected MoDCs (Fig. 5b and Supplementary Data 6 and 8 ).

We also found that the transferrin receptor TFRC was upregulated in STM-D23580-infected cells compared to both uninfected and STM-LT2 infected. This correlates with increased expression of DNAJC13, a protein that promotes recycling of transferrin directly from early endosome to plasma membrane ${ }^{31}$ (Fig. 5b and Supplementary Data 6). Since iron is a critical nutrient resource that influences intracellular bacterial growth and virulence factor expression, these data suggest that invasive Salmonella usurps iron intake mechanisms to facilitate its intracellular survival and replication ${ }^{32,33}$. 


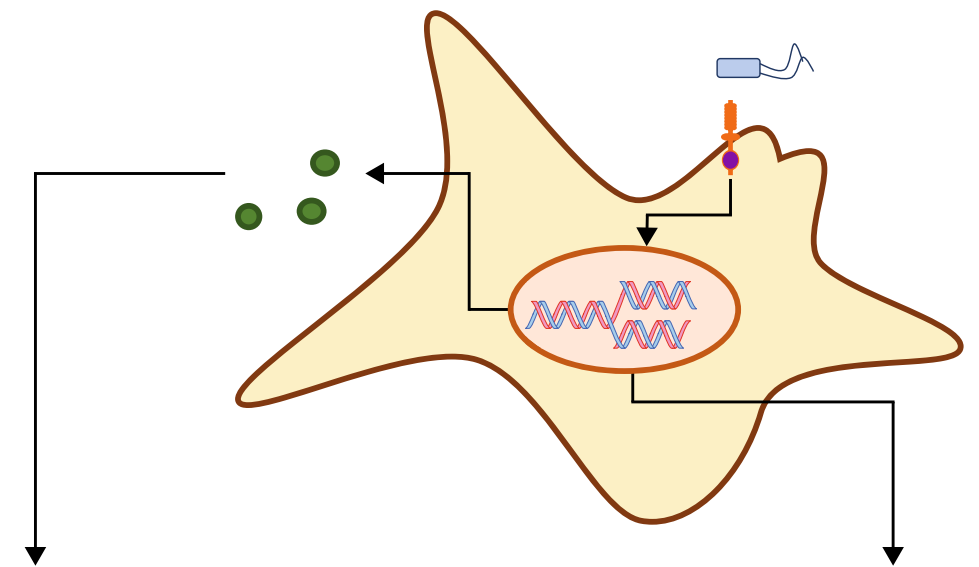

Cytokines

Transcription factors

a
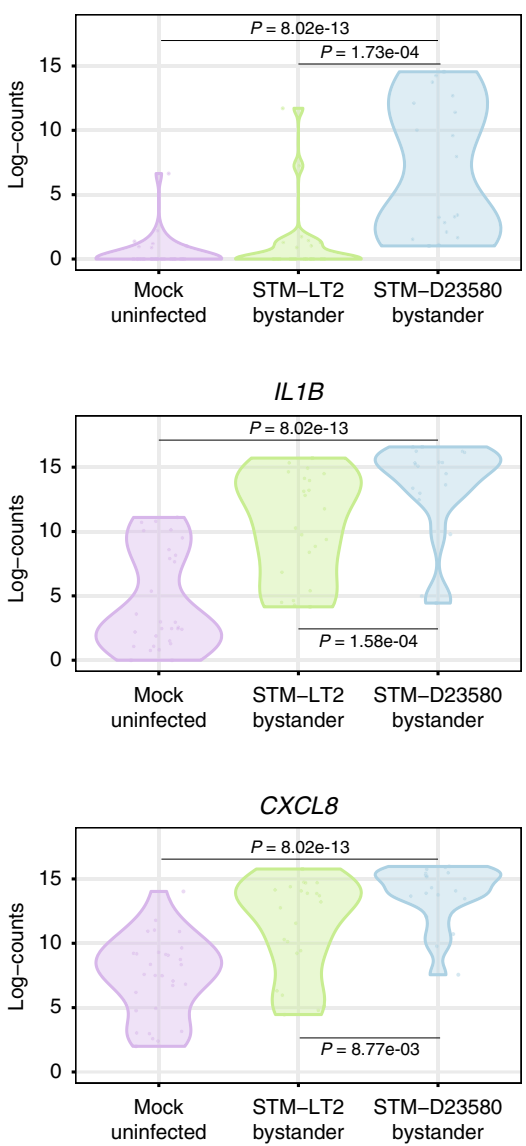

b

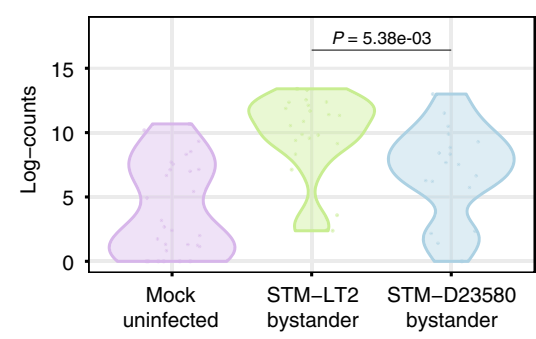

ATF3
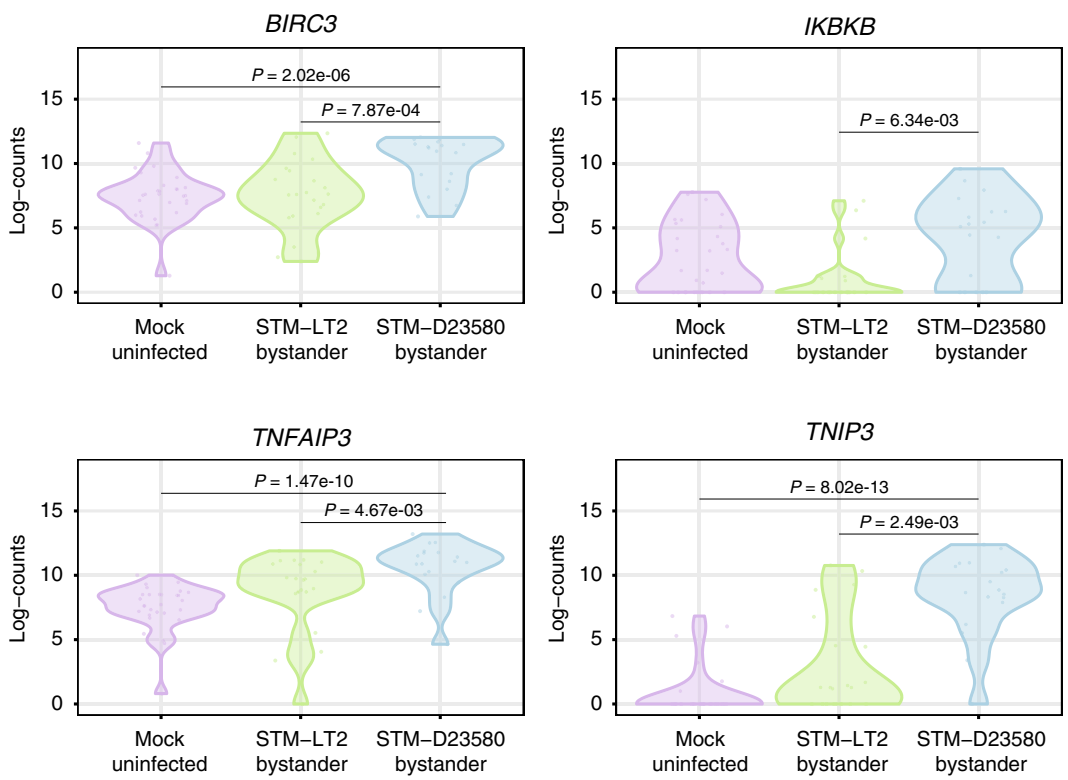

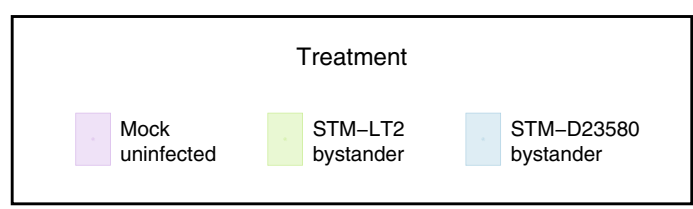

Fig. 4 STM-D23580 bystander MoDCs show an exaggerated pro-inflammatory response. a Pro-inflammatory $\operatorname{cytokines} I L 12 B^{\left({ }^{*}\right)}, I L 1 B^{(*)}$ and $C X C L 8^{\left({ }^{*}\right)}$ and

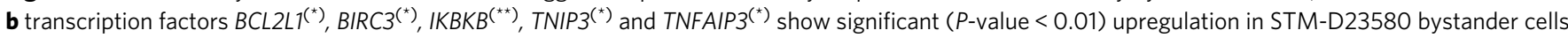
compared to both STM-LT2 bystander MoDCs and uninfected control ${ }^{(*)}$ or compared to STM-LT2 bystander MoDCs only $\left.{ }^{(\star}\right)$. ATF3 shows significant downregulation in STM-D23580 bystander cells compared to STM-LT2 bystander MoDCs 
a

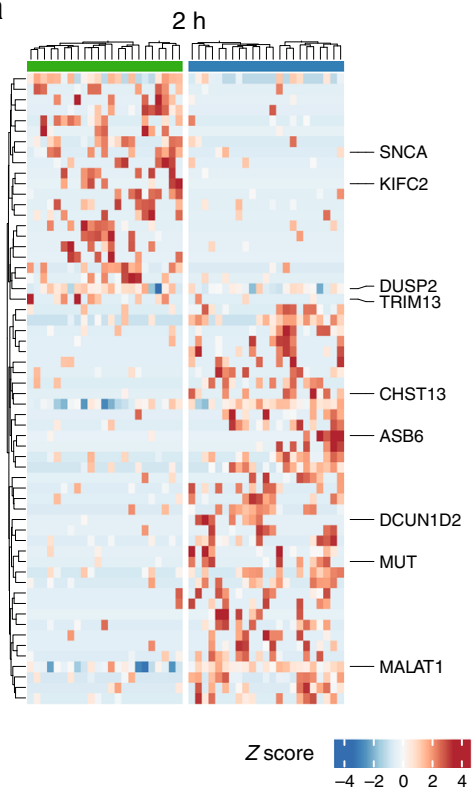

b

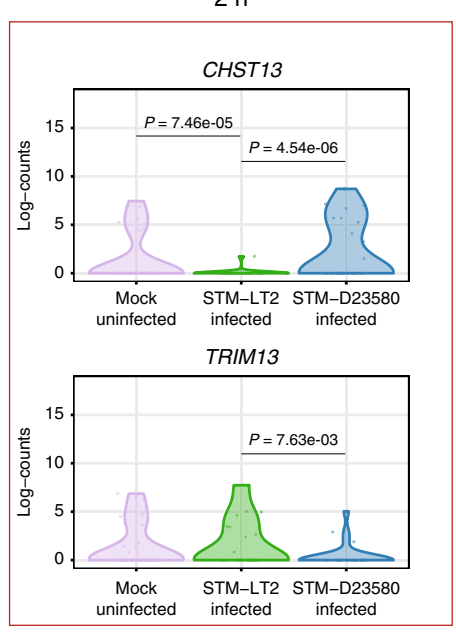

Treatment

Mock uninfected

STM-LT2 infected

STM-D23580 infected

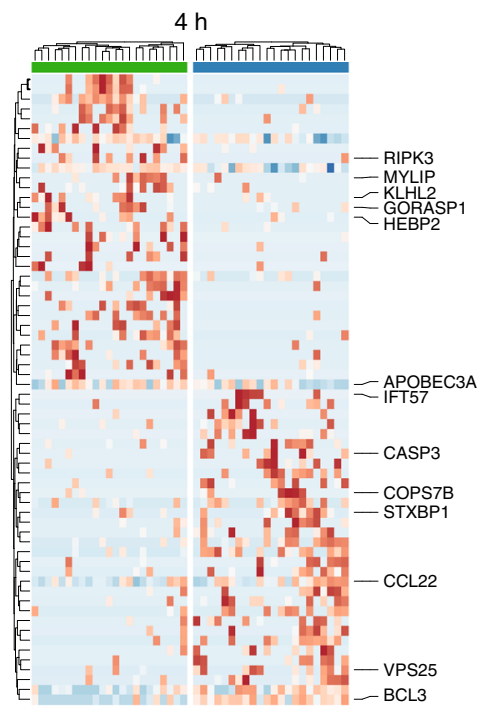

Infection $\square$ STM-LT2

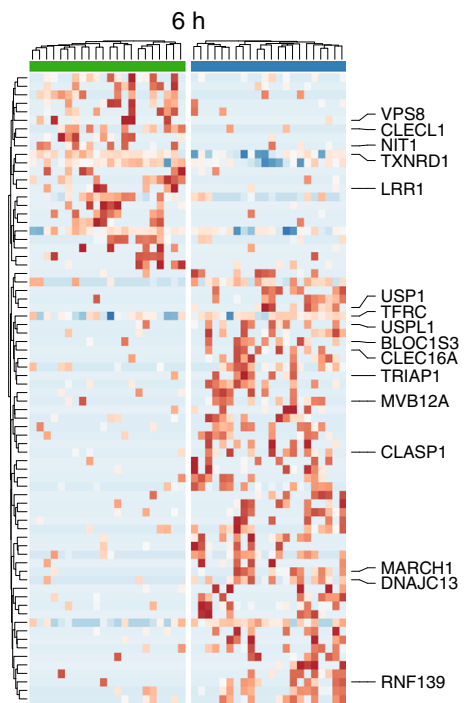

STM-D23580
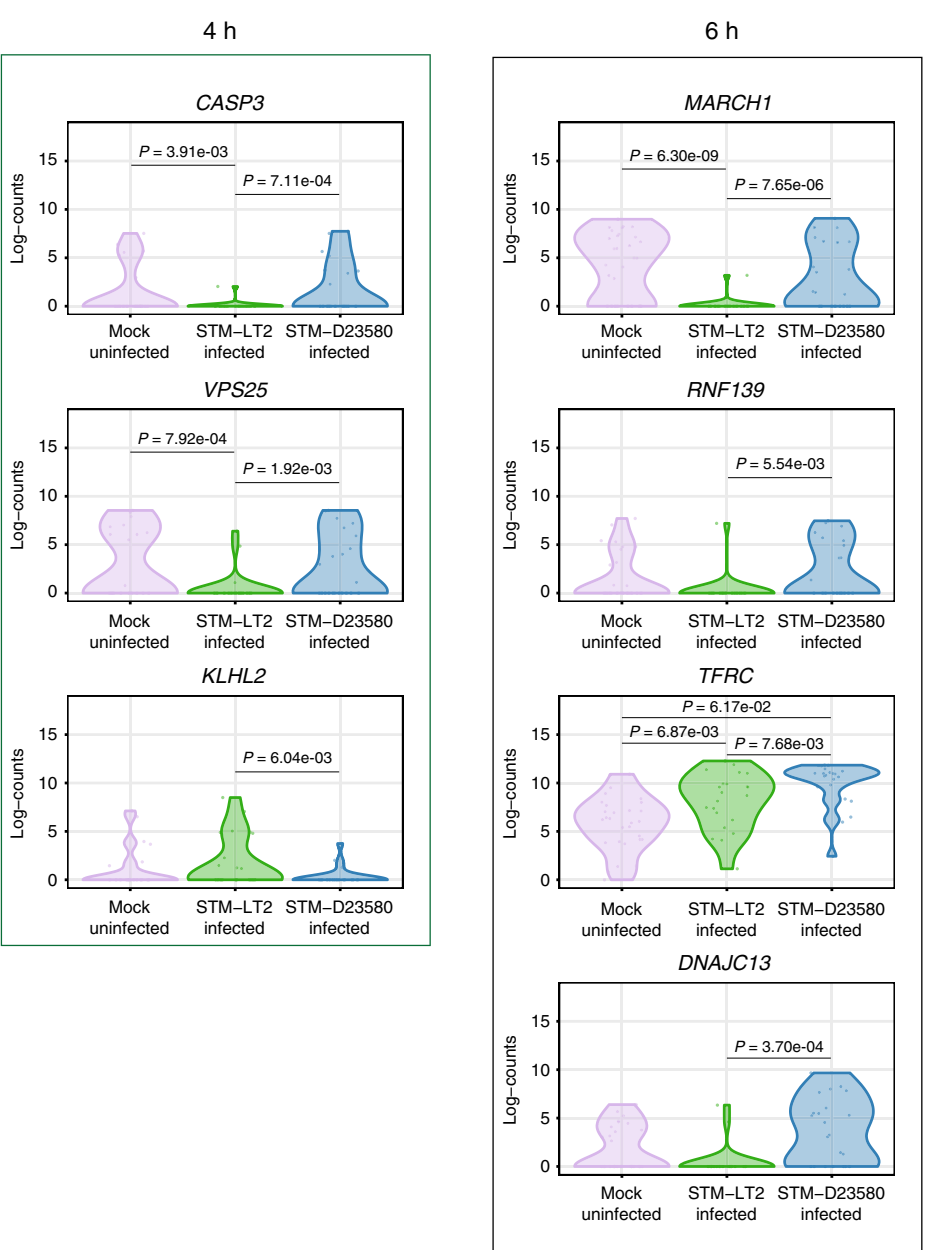

Fig. 5 Direct comparison of MoDCs infected with STM-D23580 or STM-LT2. a Heat maps displaying significant DE genes $(P$-value $<0.01)$ identified between STM-D23580 and STM-LT2-infected cells at each time point (2, 4 and $6 \mathrm{~h}$ p.i, respectively). Rows and columns were clustered using Euclidian distance and complete linkage method applied to the row-scaled values across all heat map panels at that time point. $\mathbf{b}$ Violin plots displaying the logtransformed normalized gene expression level of relevant DE genes ( $P$-value $<0.01)$ identified between STM-D23580- and STM-LT2-infected MoDCS 
STM-D23580 exploits the IL10/MARCH1/CD83 axis. Intracellular Salmonella can subvert MoDC functions by manipulating cell-intrinsic host immune pathways ${ }^{34,35}$. Consistent with this notion, we discovered that cells infected with STM-D23580 expressed a significantly higher level of IL10 and JAK2 relative to uninfected MoDCs at $6 \mathrm{~h}$ p.i. (Fig. 6a). We also found that CLECL1, involved in T cell activation and co-stimulation ${ }^{36}$, and CLEC7A, implicated in pattern recognition of pathogens leading to the activation of pro-inflammatory cytokines $\mathrm{s}^{37}$, were both significantly downregulated at $6 \mathrm{~h}$ p.i. in STM-D23580-infected cells compared to both unstimulated MoDCs and STM-LT2infected MoDCs (Fig. 6a). These data indicate that a suppressive phenotype develops in STM-D23580-infected MoDCs at later stages of infection.

MoDC maturation is also marked by the induction of the surface activation marker CD83, in addition to MHC-II and CD86. No significant differences were observed in CD83 expression among infected and bystander STM-LT2 challenged MoDCs. However, MoDCs infected with invasive Salmonella showed a bimodal expression of CD83, with a subset of cells failing to upregulate this receptor, both at the transcriptional and the protein level (Fig. 6b, c). Using flow cytometry, we confirmed a statistically significant reduction of CD83 surface expression in STM-D23580 relative to STM-LT2-infected MoDCs (Fig. 6d). MARCH1 is an E3-ubiquitin ligase which targets MHC-II and $\mathrm{CD} 86$ for degradation ${ }^{38,39}$. Upon MoDC activation, upregulation of CD83 is associated with a decrease in MARCH1 mRNA expression $^{40}$. Other studies demonstrated that the antiinflammatory cytokine IL-10 induces MARCH1 ${ }^{41,42}$. We detected significantly higher amounts of IL-10 in the supernatants of MoDCs challenged with STM-D23580 as compared to STM-LT2 at later stage of the infection (Supplementary Figure 10). This correlated with higher expression of MARCH1 in STM-D23580infected cells compared to STM-LT2 infected (Fig. 7a). The lack of downregulation of MARCH1 in STM-D23580-infected cells was confirmed by qPCR (Fig. 7b).

To investigate whether intracellular STM-D23580 could affect the antigen presentation machinery by acting through the IL10/ CD83/MARCH1 axis, we analysed surface expression of HLA-DR and CD86 on infected MoDCs by FACS. We observed that intracellular STM-D23580 correlated with a significant reduction in surface HLA-DR and CD86 expression compared with STMLT2 (Fig. 7c). In contrast, there was no difference in the surface expression of HLA-I, a known target of MARCH $9^{43}$ and CD1a, which traffics independently of MHC-II during MoDC maturation $^{44}$. These results suggest that invasive Salmonella develops molecular mechanisms to specifically target MHC-II molecules (Supplementary Figure 11).

Interestingly, we detected decreased expression of CD1a, necessary to present lipid antigens ${ }^{45}$, at the cell surface on infected cells compared to unstimulated and bystander cells (Supplementary Figure 12A), consistent with our single-cell data (Supplementary Figure 12B). On the contrary HLA-DR and HLA-I surface expression were upregulated in infected MoDCs compared to both bystander or uninfected cells.

STM-D23580 impairs antigen presentation to $\mathrm{CD4}^{+} \mathrm{T}$ cells. Considering the role of $\mathrm{CD} 83$ in $\mathrm{T}$ cell stimulation ${ }^{46}$, we tested whether reduced CD83 surface expression on infected MoDCs correlated with diminished $\mathrm{T}$ cell stimulatory capacity. We developed an in vitro antigen presentation assay where Salmonella infected MoDCs were co-cultured with $\mathrm{CD} 4^{+} \mathrm{T}$ cell clones specific for PhoN ${ }^{47}$, a protein expressed in multiple Salmonella serovars, including $S$. Typhimurium. At $6 \mathrm{~h}$ p.i., we FACS sorted infected cells based on their CD83 surface expression. While
STM-LT2 infected MoDCs were all CD83+ (Fig. 8a and Supplementary Figure 13A), STM-D23580-infected MoDCs could be separated into CD83-llow and CD83 high cells (Fig. 8b and Supplementary Figure 13B). Uninfected MoDCs were used as $\mathrm{CD}^{- \text {-low }}$ control (Fig. 8c and Supplementary Figure 13D). We then co-cultured the sorted MoDCs with PhoN-specific T cell clones and tested them for their ability to secrete IFN- $\gamma$ and TNFa, by intracellular cytokine staining. STM-LT2 and STM-D23580 $\mathrm{CD} 83^{\text {high }}$ infected MoDCs triggered $\mathrm{CD} 4^{+} \mathrm{T}$ cell activation, with production of TNF- $\alpha$ and IFN- $\gamma$. In contrast, T cells co-cultured with STM-D23580 CD83-/low infected MoDCs produced significantly lower levels of these cytokines (Fig. 8d,e and Supplementary Figure 13E).

We corroborated the specificity of our assay using $\mathrm{CD} 4^{+} \mathrm{T}$ cell clones recognizing HlyE, a protein expressed in $S$. Typhi but not $S$. Typhimurium ${ }^{47,48}$. As expected, HlyE-specific $\mathrm{CD} 4^{+} \mathrm{T}$ cell clones responded exclusively to CD83 ${ }^{+}$ST-Ty2-infected MoDCs (Supplementary Figure 13C and 13E). In addition to cytokine production, our co-culture model indicates that invasive Salmonella may also affect $\mathrm{T}$ cell costimulatory signals, such as the engagement of CD40 with CD40L. In comparison to CD83high STM-D23580-infected cells, we observed a reduction of $\mathrm{CD} 40 \mathrm{~L}$ expression among cytokine-producing $\mathrm{T}$ cell clones co-cultured with CD83-/low STM-D23580-infected MoDCs (Fig. 8f, g), suggesting that the suboptimal activation of $\mathrm{T}$ cells in the presence of invasive Salmonella might influence also the expression of costimulatory molecules on T cells. Together, these data indicate that the intracellular activity of invasive Salmonella, in combination with reduced CD83, may impair the ability of MoDC subsets to trigger antigen-specific $\mathrm{T}$ cell activation.

Small bulks recapitulate average RNA-sequencing profiles. To validate our scRNA-seq findings, we conducted bulk RNA-seq of 5000 cells from infected and bystander MoDCs treated with either invasive or non-invasive Salmonella using a modified version of the Smart-seq2 protocol. Principal component analysis (PCA) of these bulk samples revealed a distribution of the various experimental conditions comparable to the diffusion map representation of our single-cell data. These results confirm the distinction between uninfected and challenged MoDCs, but also bystander and infected cells (Supplementary Figure 14A). Differential expression analysis of bulk samples confirmed higher expression of CTSD, LGMN, CCL7, CCL26, MS4A4A, LAMP1 and APOE in infected cells, and IL1B, PLAT, CD1C, CD1A, CCL1, TRAF4 and CD83 in bystander cells (Supplementary Figure 14B and Supplementary Data 9). We also confirmed that MARCH1 expression in STM-D23580-infected cells was comparable to uninfected cells, which contrasted with a marked downregulation of MARCH1 expression in STM-LT2-infected MoDCs. In addition, we confirmed that CD83 and IL10 expression was differentially regulated between STM-LT2- and STM-D23580-infected cells, although the bulk method failed to resolve the cell-to-cell variability detected only with scRNA-seq (Supplementary Figure 14C).

Independent validation using QPCR. We validated key genes identified as differentially expressed between infected and bystander MoDCs using qPCR. First, we identified candidate markers from genes identified both in our previous analyses of unsupervised clusters and direct comparisons (i.e., infected relative to bystander MoDCs or STM-D23580 relative to STM-LT2). After FACS sorting and cDNA preparation according to the Smart-seq2 protocol, we then pooled cDNA from individual cells from each group and performed qPCR for selected genes. Statistical analysis of the relationship between our $\mathrm{QPCR}$ and 
a

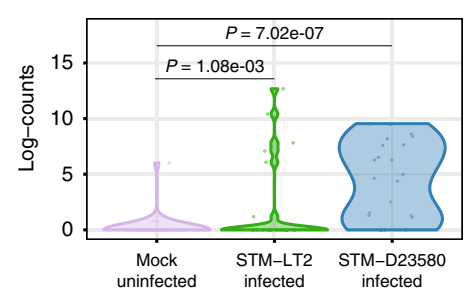

JAK2

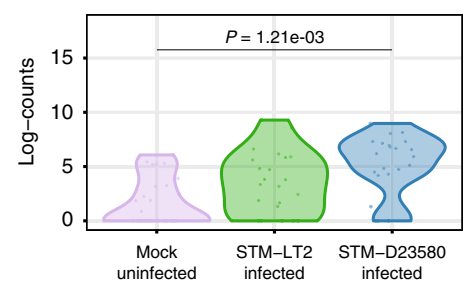

CLECL1

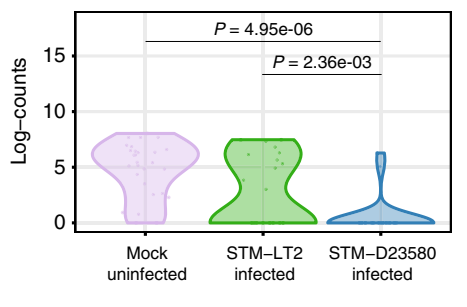

CLEC7A

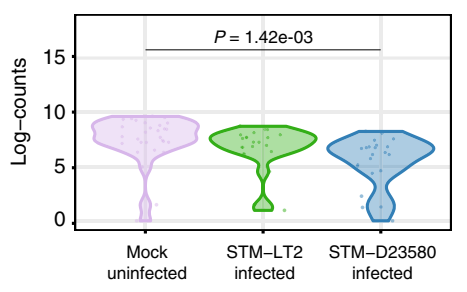

b
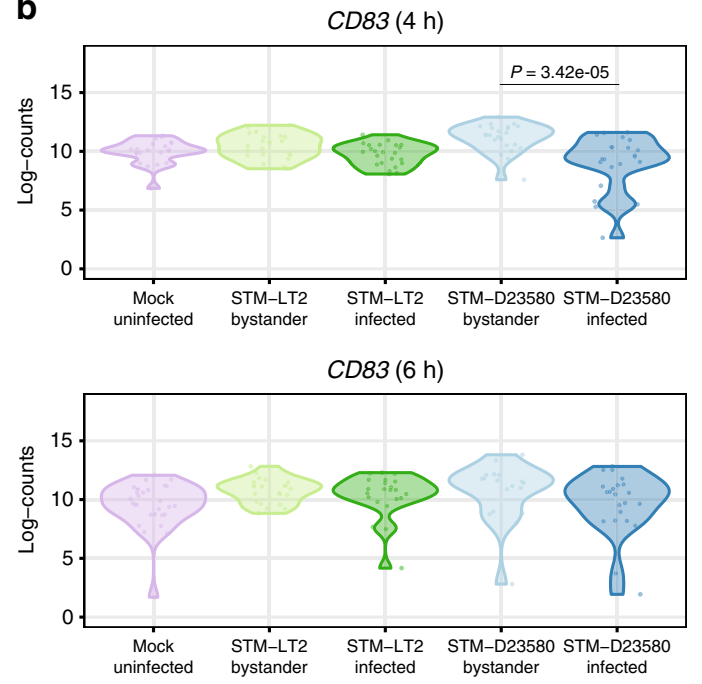

C

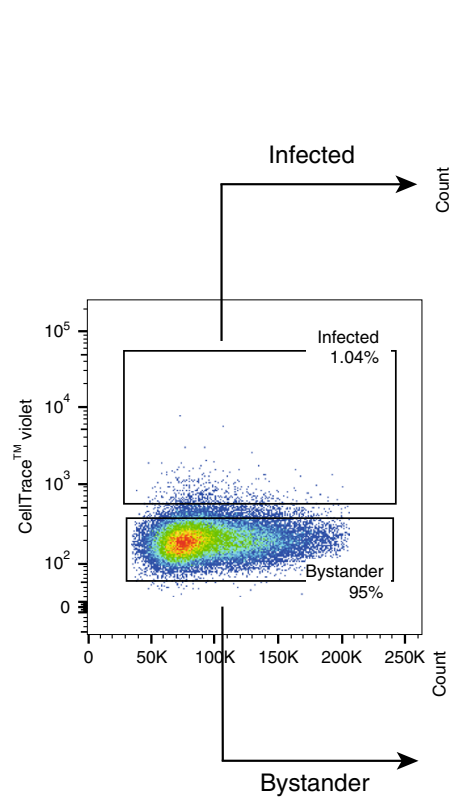

Mock

STM-LT2

STM-D23580

d

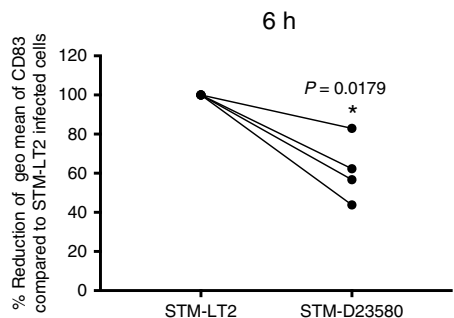

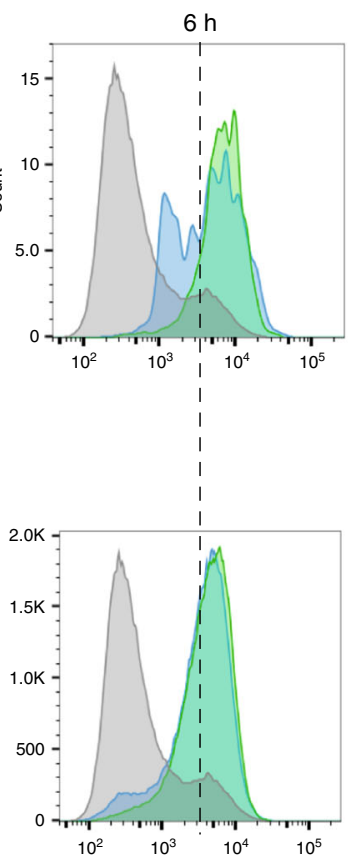

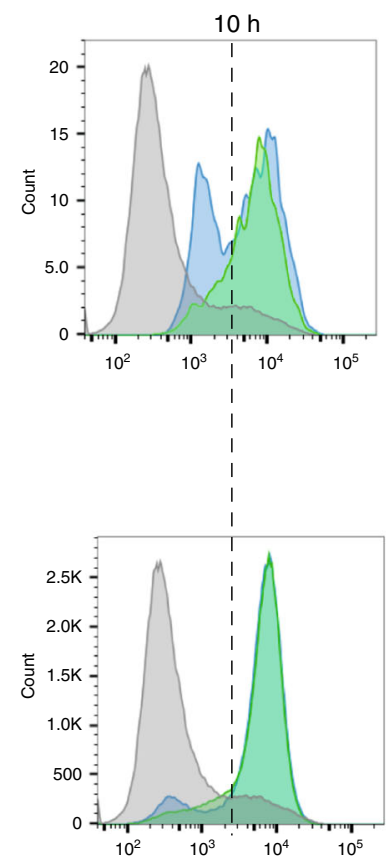

CD83 - PE

$10 \mathrm{~h}$

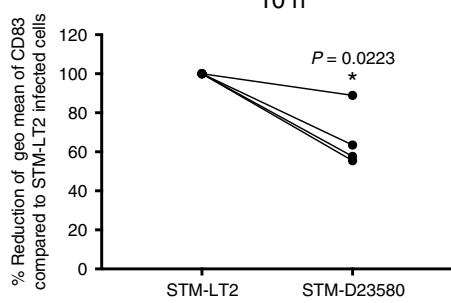

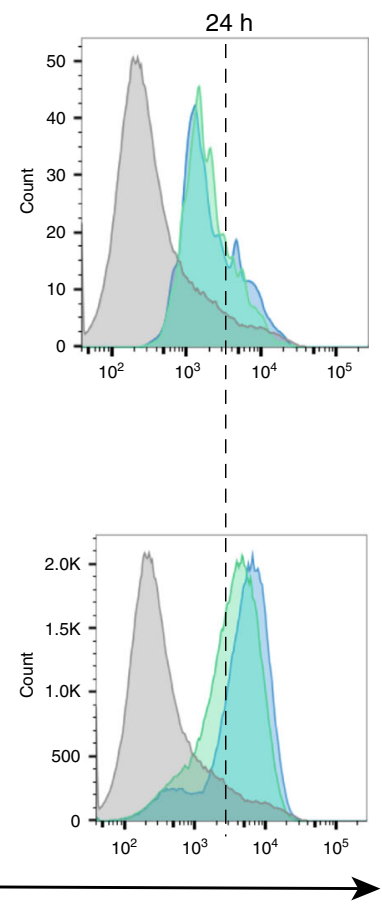

$24 \mathrm{~h}$

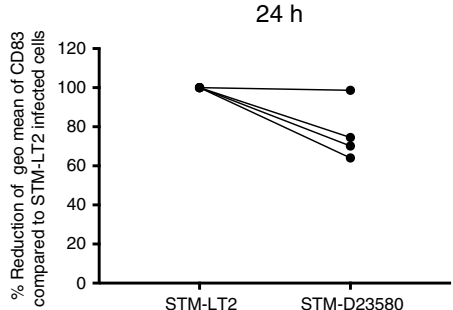

Fig. 6 Intracellular STM-D23580 reduces CD83 surface expression. a Violin plots showing single-cell gene expression of IL10, JAK2, CLECL1 and CLEC7A at $6 \mathrm{~h}$ p.i. in infected and uninfected cells. b Violin plots showing single-cell gene expression of CD83 at 4 and $6 \mathrm{~h}$ p.i. in all experimental groups. c Flow cytometry histograms showing the expression of surface CD83 on gated infected and bystander cells challenged with STM-D23580 (blue), STM-LT2 (green) or left uninfected (grey) for 6, 10 or $24 \mathrm{~h}$. STM-D23580-infected MoDCs display decreased surface expression of CD83 as compared with STMLT2. Histograms from one representative example of at least four biological replicates. d STM-D23580 infection reduces surface CD83 levels detected by flow cytometry. On the $y$-axis, the geometric mean of fluorescence levels for CD83 on STM-D23580-infected cells is represented as a percentage of the level in STM-LT2-infected cells, set at 100\%. Four independent experiments are shown. Two-tailed paired Student's $t$-test, $P$-value $<0.05\left({ }^{*}\right)$ 
a

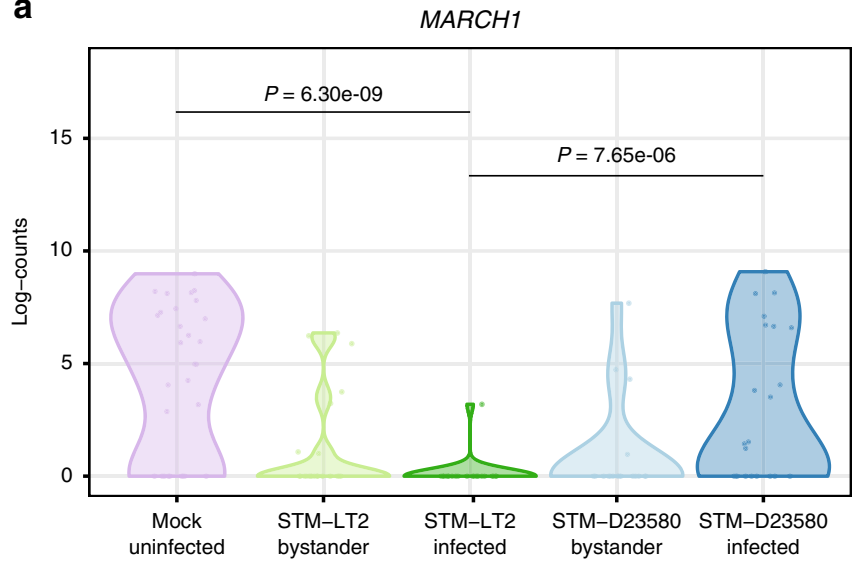

b

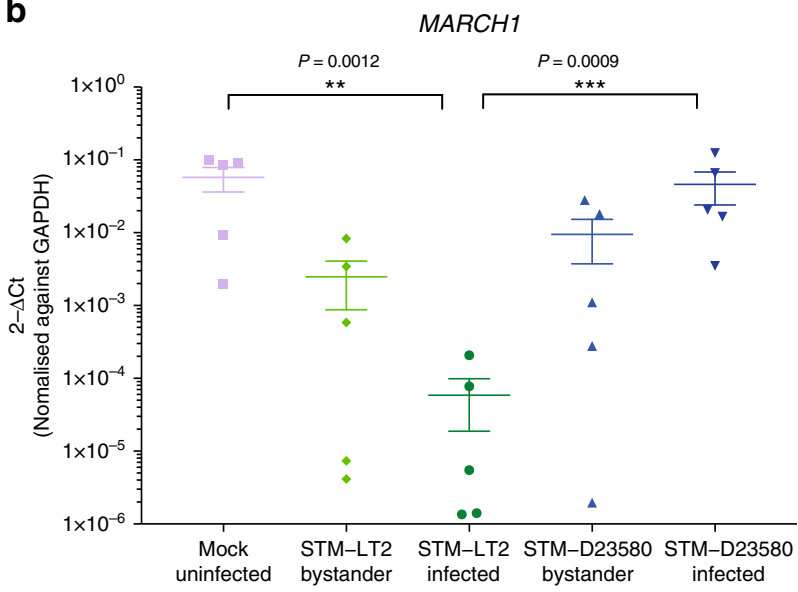

C
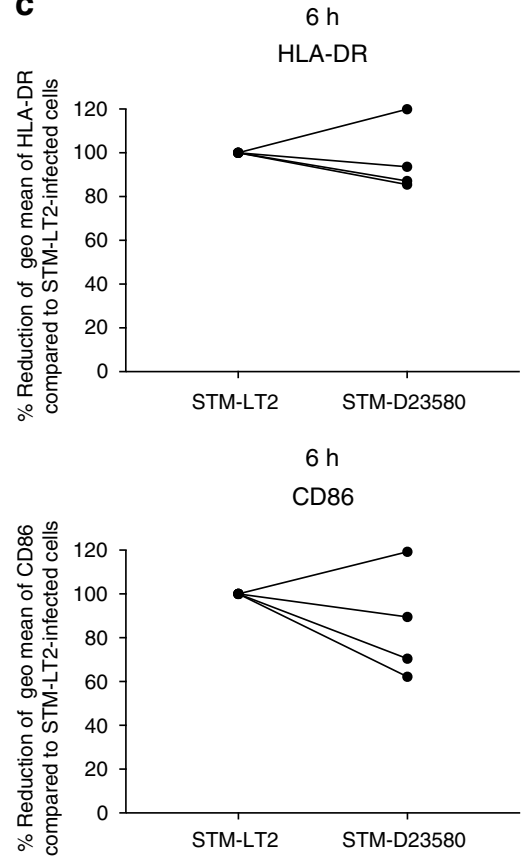

$10 \mathrm{~h}$
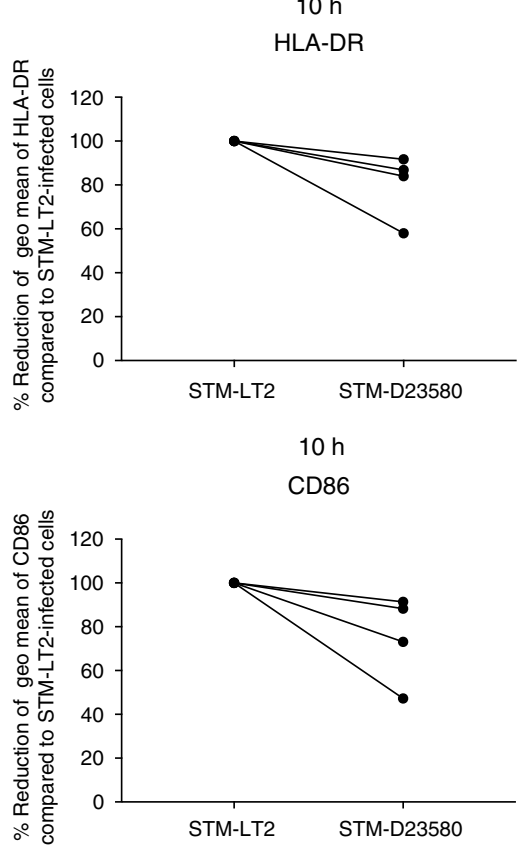

$24 \mathrm{~h}$

HLA-DR
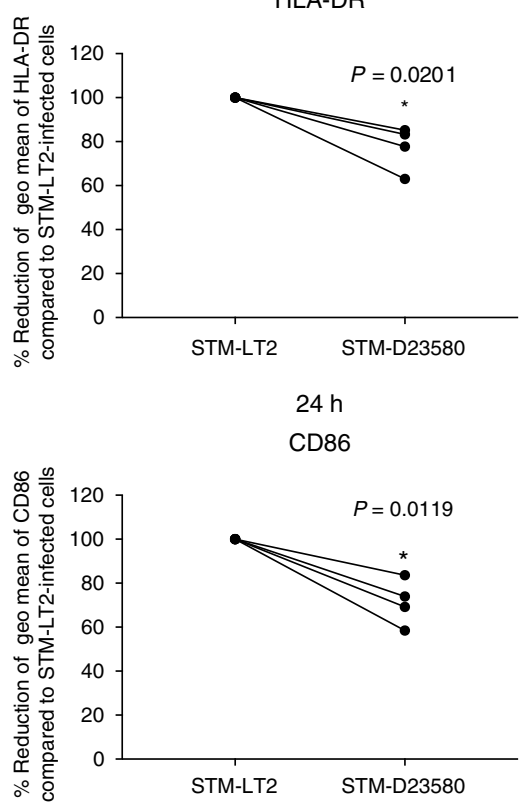

Fig. 7 Intracellular STM-D23580 reduces HLA-DR and CD86 surface expression compared to STM-LT2. a Violin plot showing single-cell gene expression of $M A R C H 1$ at $6 \mathrm{~h}$ p.i. in all the experimental groups. $\mathbf{b}$ Dot plot showing gene expression of $M A R C H 1$ at $6 \mathrm{~h}$ p.i detected by qPCR in all experimental groups. The mean \pm SEM of five independent experiments is shown. Two-way ANOVA test, $P$-value $<0.01\left(^{\star \star}\right),<0.001\left(^{\star \star \star}\right)$. c Surface HLA-DR and CD86 expression measured by flow cytometry. On the $y$-axis, the geometric mean of fluorescence levels for HLA-DR and CD86 on STM-D23580-infected cells is represented as a percentage of the level in STM-LT2 infected cells, set at 100\%. Four independent experiments are shown. Two-tail paired Student's t-test, $P$-value $<0.05\left(^{\star}\right)$

scRNA-seq results revealed a significant correlation between the two methods across all genes tested (Supplementary Figure 15). This analysis confirmed that infected cells induced CTSL, CTSD and $M S A 4 A 4 A$, while bystander cells predominantly expressed PLAT, EBI3 and STAT5B. We also validated MARCH1 as a marker of STM-D23580-infected MoDCs and VPS25 as a marker for the STM-LT2 infected population (Supplementary Figure 15).

\section{Discussion}

Here we define how invasive Salmonella STM-D23580 directs divergent and heightened heterogeneity of single-cell profiles of infected and bystander MoDCs compared to non-invasive STMLT2. Specifically, invasive Salmonella-containing MoDCs differentially regulated genes associated with endosomal trafficking and antigen presentation pathways. Invasive Salmonella also induced IL10 and downregulated CD83 likely via its inability to downregulate $M A R C H 1$. These mechanisms evoke suboptimal signals for efficient CD4 ${ }^{+} \mathrm{T}$ cell activation. MoDCs exposed to, but not harbouring replication-competent invasive Salmonella, expressed increased levels of pro-inflammatory transcription factors and cytokines. We then validated these results by both resequencing sorted subsets, and at mRNA and protein levels in independent donors. In summary, our scRNA-seq analyses reported here reveal hyper-activation of bystander MoDCs and mechanisms of cell-intrinsic host adaption exploited by Salmonella ST313 with implications for its invasive behaviour in immunocompromised hosts.

Heightened activation of bystander MoDCs following challenge with invasive Salmonella may facilitate recruitment of adaptive immune cells to non-infected rather than infected MoDCs and serve a putative decoy role. Divergent responses in bystander MoDCs may arise in part from differences in the PAMP composition of the STM-D23580 surface coat, since treatment with of 
a
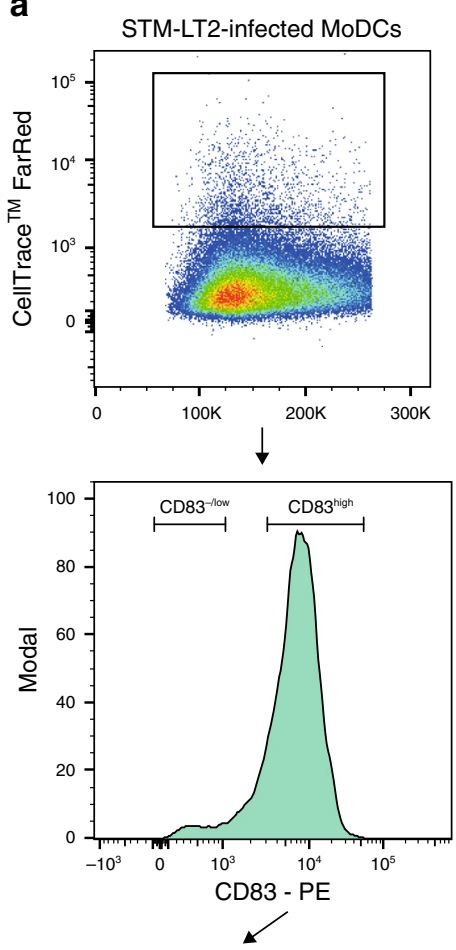

b
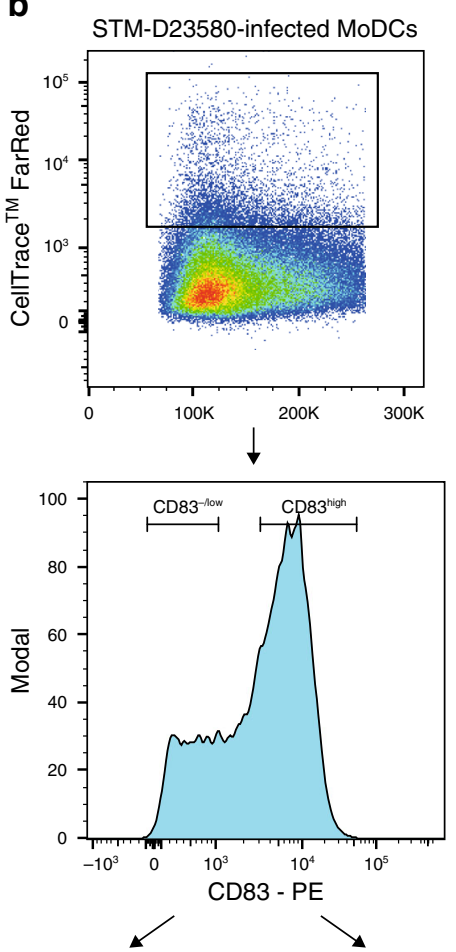

C
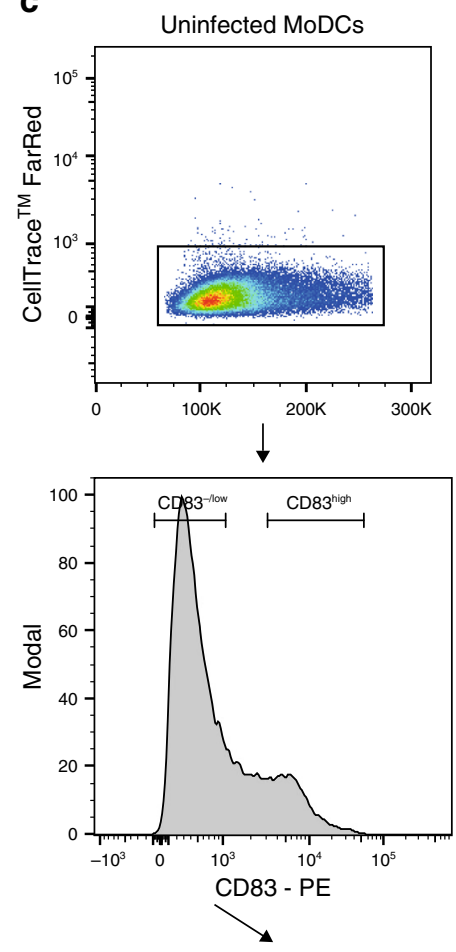

d CD4+ T cells
+ CD83 $3^{+}$STM-LT2-infected
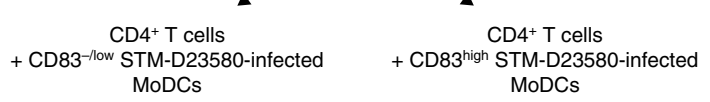

uninfected MoDCs
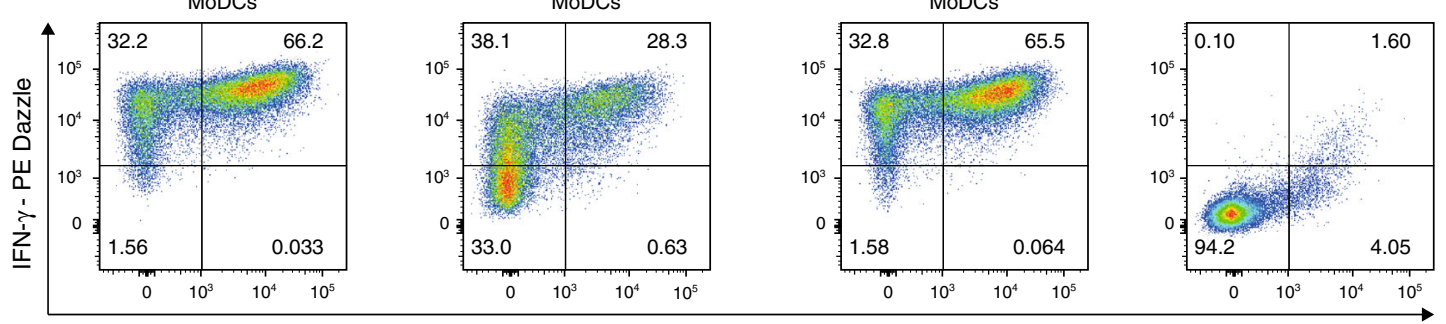

TNF- $\alpha-$ PECy7
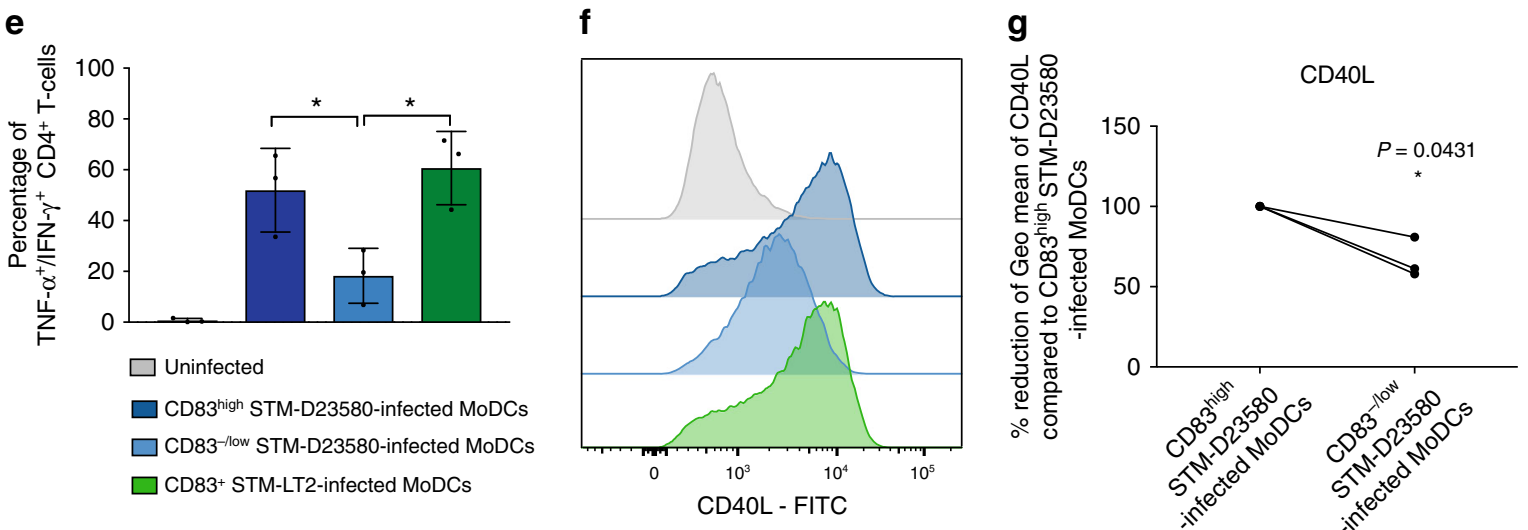

Fig. 8 CD83-/low STM-D23580-infected MoDCs direct defective activation of Salmonella-specific CD4+ T cells. At 6 h p.i., a STM-LT2-infected MoDCs were sorted as $\mathrm{CD}_{83}{ }^{+}$while $\mathbf{b}$ STM-D23580-infected MoDCs were sorted as CD83-/low or CD83 ${ }^{\text {high }}$. $\mathbf{c}$ Uninfected MoDCs were used as negative control. Sorted subsets of infected MoDCs were co-cultured overnight with PhoN-specific CD4 ${ }^{+} \mathrm{T}$ cell clones cross-reactive against typhoidal and non-typhoidal serovars. $\mathbf{d}$ Capacity of CD4 ${ }^{+}$T cell to secrete IFN- $\gamma$ and TNF- $\alpha$, as detected by intracellular staining. FACS plots show the percentage of IFN- $\gamma^{+} / \mathrm{TNF}-\alpha^{+}$ cells out of total $\mathrm{CD}^{+} \mathrm{CD}^{+} \mathrm{T}$ cells. Dot plots comprise a representative experiment out of four. e CD83-/low STM-D23580-infected MoDCs induce a significantly lower amount of cytokine-producing T cells, as compared to CD83 high STM-D23580 or CD83+ STM-LT2 infected cells. The mean \pm SEM of three independent experiments is shown One-way ANOVA test, $P$-value $<0.05\left(^{*}\right)$. f Flow cytometry histogram showing CD4OL expression on T cells cocultured with $\mathrm{CD}_{3} 3^{+}$STM-LT2 infected MoDCs, CD83-/low or CD83 high STM-D23580-infected cells. Histograms from one representative example of at least three biological replicates. $\mathbf{g}$ Geometric mean of fluorescence intensity for CD40L in T cells co-cultured with CD83-/low STM-D23580-infected MoDCs, represented as a percentage of reduction from CD83 high STM-D23580-infected MoDCs. three independent experiments are shown. Two-tailed paired Student's $t$-test, $P$-value $<0.05\left(^{\star}\right)$ 
DNAase, RNAase and proteinase $\mathrm{K}$ did not impair its immunostimulatory ability. We also observed widespread changes in expression levels of key transcription factors in MoDCs exposed to STM-D23580 with corresponding induction of proinflammatory cytokines, such as IL-12p40 and IL-1 $\beta$.

Cells that internalized STM-D23580 displayed reduced maturation and downregulated components of the antigen presentation machinery, likely via induction of IL10 and dysregulated expression of MARCH1. IL10 induction as an evasion strategy by STM-D23580 is in line with previous observations that explain how increased IL-10 expression enhances susceptibility to invasive NTS infection and blunts intestinal inflammation $^{49}$. Consistent with inducing IL-10, both at transcriptional and protein level, STM-D23580 also induced MARCH1. MARCH1 is an E3 ubiquitin ligase that plays an important role in ubiquitination and degradation of MHC-II and CD86 $38,39,50$. In particular, the enzymatic function of MARCH1 towards MHC-II and CD86 only occurs in the absence of appreciable levels of $\mathrm{CD}^{4} 3^{40}$, an immunoglobulin-like surface molecule that normally blocks MHC-II/CD86 association with MARCH1. Differential expression of the IL10/CD83/MARCH1 axis by invasive Salmonella represents a coordinated strategy employed by this strain to evade effective antigen presentation in cells harbouring live bacteria. To our knowledge, this is the first description of such an evasion mechanism by this pathovar.

We propose that invasive Salmonella impairs the ability of cells to present antigen to $\mathrm{CD} 4^{+}$restricted $\mathrm{T}$ cells by reducing surface MHC-II. Our proposal agrees with studies showing that infection with Salmonella inhibits antigen presentation by murine $\mathrm{MoDCs}^{51}$. Here, we employed PhoN-specific CD4 ${ }^{+} \mathrm{T}$ cells clones generated from volunteers diagnosed with enteric fever ${ }^{47}$. We observed that the subset of STM-D23580-infected MoDCs with CD83-/low expression showed significant defects in their ability to elicit production of TNF- $\alpha$ and IFN- $\gamma$ by CD4 ${ }^{+}$T cells. The defective $\mathrm{T}$ cell costimulatory capacity of $\mathrm{CD} 83^{-/ \mathrm{low}}$ STMD23580-infected MoDCs is consistent with the observation that specific siRNA-mediated knockdown of CD83 has a marked effect on the capacity of MoDCs to stimulate T cell responses ${ }^{46}$. MoDCs infected with the Opa ${ }_{\mathrm{CEA}}$-expressing strain of Neisseria gonorrhoeae did not display any surface expression of CD83 and showed a reduced capacity to stimulate an allogeneic $\mathrm{T}$ cell proliferative response $\mathrm{s}^{52}$. Other pathogens that establish latency, such as herpes simplex virus type 1 (HSV-1), human cytomegalovirus (HCMV) and vaccinia virus, adopt different strategies to interfere with CD83 expression on the surface of infected MoDCs. This process prevents MoDC-mediated T cell activation and proliferation ${ }^{53-56}$. For example, HCMV causes either CD83 degradation in a proteasome-dependent manner or shedding from the surface of mature MoDCs ${ }^{56,57}$. HSV-1 induces both mRNA and protein degradation of CD83 in mature MoDCs $53,55,58$. Intriguingly, surface expression of CD83 appears downregulated only by the ST313 Salmonella strain, while both STM-LT2 and the typhoidal strain Ty2 show comparable CD83 levels.

A positive regulator like CD40L when associated with CD40 not only activates T cells, but also activates MoDCs. Pathogens can downregulate CD40 expression on APCs. For instance, Helicobacter pylori reduces the expression of CD40L on T cells, exploiting the CD40/CD40L pathway for its survival ${ }^{59}$. Bordetella pertussis and Bordetella bronchiseptica both decrease surface levels of CD40 on MoDCs to promote development of regulatory $\mathrm{T}$ cells ${ }^{60,61}$. A significant role for CD40/CD40L interactions exists to mount a successful cellular immune response against Salmonella in normal BALB/c mice ${ }^{62}$. In addition, MHC-II, CD80, CD86 and CD40 are downregulated during chronic infection with $\mathrm{BCG}^{63}$ and both in in vitro and lepromatous patients by
Mycobacterium leprae ${ }^{64,65}$. Co-stimulation not only amplifies the magnitude of T cell and APC interaction, but also fine-tunes the downstream immune signalling.

Studies using scRNA-seq to map host responses to Salmonella in murine macrophages revealed that variable PhoP/PhoQ activity leading to LPS modification underlies this heterogeneity in host response ${ }^{66}$. Macrophages can induce either alternative pro-inflammatory M1-like or anti-inflammatory M2-like maturation states, depending on the replication status of Salmonella ${ }^{16}$. So far, studies identified three subpopulations of infected macrophages corresponding to consecutive stages of infection ${ }^{67}$. Our work extends this heterogeneity in cell-to-cell response to human MoDCs. Our results also demonstrate that scRNA-seq can uncover subtle differences in host-pathogen interactions between genetically similar Salmonella strains.

A recent study demonstrated that $\mathrm{CD} 11 \mathrm{~b}^{+}$migratory DCs facilitate STM-D23580 hyper-dissemination to systemic sites in vivo ${ }^{68}$. The scRNA-seq analysis presented here elucidates fine mechanisms by which the invasive STM-D23580 strain manipulates gene expression of infected and bystander MoDCs. We propose that downregulation of costimulatory pathways by invasive Salmonella results in a response detrimental to the host. Since any negative effect on antigen presentation can further accentuate inherent $\mathrm{CD}^{+}{ }^{+} \mathrm{T}$ cell defects observed in immunocompromised individuals, our results delineate a possible molecular pathway exploited by ST313 to evade immediate detection. Overall, these observations contribute to a better understanding of the pathogenesis and dissemination of invasive NTS disease.

\section{Methods}

Bacterial strains and labelling. Salmonella enterica serovar Typhimurium (STM) strain LT2 (ATCC 700220) and the clinical isolate STM-D23580, were used as representative non-typhoidal Salmonella sequence type 19 (ST19) and type 313 (ST313), respectively. Strain LT2 is one of the principal Salmonella laboratory strains used in cellular and molecular biology ${ }^{69}$. Strain D23580 was isolated from the blood of an HIV-negative Malawian child with malaria and anaemia. Salmonella enterica serovar Typhi (ST) strain Ty2 (ATCC 700931) was used as representative typhoidal serovar. All bacteria were grown to logarithmic growth phase in LB Lennox broth (Sigma) supplemented with sucrose (Sigma) at a final concentration of $10 \%$. Aliquots were kept frozen at $-80^{\circ} \mathrm{C}$ until use, while bacterial viability was monitored periodically. For each experiment, an aliquot of bacteria was thawed, diluted in RPMI 1640 (Sigma) and incubated in the presence of 5uM of CellTrace ${ }^{\mathrm{TM}}$ Violet or CellTrace ${ }^{\mathrm{TM}}$ Far Red Cell Proliferation kit (ThermoFisher) at $37^{\circ} \mathrm{C}$ for $20 \mathrm{~min}$ while shaking $(200 \mathrm{rpm})$. Bacteria were then washed and re-suspended in RPMI to obtain a multiplicity of infection (MOI) of 10:1. The number of microorganisms was assessed at each time point post infection by plating 10-fold dilutions of the bacterial suspension, in quadruplicate, on LB Lennox agar (Sigma). The number of bacteria was determined as colony forming units (CFU)

Preparation of heat-killed bacteria. Salmonella strains were grown in LB broth supplemented with $10 \%$ sucrose. Bacteria were harvested during the logarithmic growth phase, diluted to an OD 0.5 , and killed by heating at $100^{\circ} \mathrm{C}$ for $15 \mathrm{~min}$. The suspension of heat-killed $(\mathrm{HK})$ bacteria was allowed to cool at room temperature (RT) for $15 \mathrm{~min}$ and, subsequently, plated on LB agar to confirm bacteria were no longer viable. Enzymatic digestion of nucleic acid was performed using $10 \mathrm{mg} / \mathrm{mL}$ of DNase I and RNase (Qiagen) for $30 \mathrm{~min}$ at $37^{\circ} \mathrm{C}$, followed by $10 \mathrm{mg} / \mathrm{mL}$ of Proteinase K (Qiagen) at $59^{\circ} \mathrm{C}$ for $3 \mathrm{~h}$. Decreasing amount $(50-1 \mu \mathrm{L})$ of the $\mathrm{HK}$ bacterial suspensions were used to stimulate MoDCs. LPS from $S$. enterica serovar Typhimurium (Sigma) was used as a control $(100-0.01 \mathrm{ng} / \mathrm{mL})$.

Generation and infection of MoDCs. Leukocyte Reduction System cones were obtained from the UK National Blood Centre with informed consent following local ethical guidelines. Blood was diluted in phosphate-buffered saline (PBS) and layered on a standard density gradient (Lymphoprep ${ }^{\mathrm{TM}}$ ). Peripheral Blood Mononuclear Cells (PBMC) were collected from the interface and washed in PBS at $4{ }^{\circ} \mathrm{C}$. Monocytes were obtained by magnetic positive selection, using the human anti-CD $14^{+}$MicroBeads (Miltenyi Biotec, Germany) according to the manufacturer's protocol.

Freshly isolated monocytes were plated in tissue culture treated dishes (Falcon) at a density of $1 \mathrm{E}+06 / \mathrm{mL}$. Differentiation into monocyte-derived dendritic cells (MoDCs) was induced in the presence of $40 \mathrm{ng} / \mathrm{mL}$ of recombinant human (rh) 
granulocyte macrophage-colony-stimulating factor (GM-CSF; PeproTech) and 40 ng/mL rh Interleukin-4 (IL-4; PeproTech).

After 5 days of culture, MoDCs were harvested, re-suspended in RPMI supplemented with $10 \%$ Fetal Bovine Serum (FBS, Sigma) and 2mM L-Glutamine (Sigma) at a density of $1 \mathrm{E}+06 / \mathrm{mL}$, and seeded $1 \mathrm{~mL} /$ tube in polypropylene tubes (Falcon). MoDCs were infected with STM-LT2 or STM-D23580 at a MOI of 10 and immediately spun down for $5 \mathrm{~min}$ to maximize bacteria-cell contact. This MOI was used, so that the frequency of infection was high enough to enable observation of infection events at the single-cell level in subsequent sorting experiments, while minimizing Salmonella-induced MoDCs death.

After incubation for $45 \mathrm{~min}$ at $37^{\circ} \mathrm{C}$, extracellular bacteria were washed away with PBS. MoDCs were then incubated for $30 \mathrm{~min}$ in RPMI supplemented with $10 \% \mathrm{FBS}, 2 \mathrm{mM}$ L-glutamine and $100 \mu \mathrm{g} / \mathrm{mL}$ of gentamycin (MP Biomedicals) to kill extracellular bacteria. Gentamycin concentration was subsequently reduced to $30 \mu \mathrm{g} / \mathrm{mL}$ for the remainder of the experiment. At each time point, cells were lysed by addition of $500 \mu \mathrm{L}$ of saponin $1 \%(\mathrm{w} / \mathrm{v})$ (Sigma) in PBS followed by $5 \mathrm{~min}$ incubation at $37^{\circ} \mathrm{C}$. Cell lysates were serially diluted 10-fold in PBS and aliquots were plated onto LB agar. The number of intracellular bacteria was determined as CFU. The uptake of the bacteria was calculated as the number of bacteria recovered at $45 \mathrm{~min}$ p.i. divided by the initial number of bacteria. The percentage of bacterial survival was calculated as the number of bacteria recovered at each time point p.i. divided by the number of bacteria recovered at $75 \mathrm{~min}$ p.i. MoDCs viability was assessed by Tripan blue exclusion assay.

Flow cytometry sorting. At 2, 4 or $6 \mathrm{~h}$ post infection (p.i.), cells were harvested, washed, stained with Propidium Iodide (Biolegend) and re-suspended in FACS buffer containing PBS, $0.3 \%(\mathrm{v} / \mathrm{v})$ bovine serum albumin (BSA, Sigma) and $2 \mathrm{mM}$ EDTA (Invitrogen). Samples were immediately acquired on a FACSAria III sorter (BD Biosciences), applying fluorescence minus one controls to adjust compensation and sorting gates. Accuracy of a high purity single-cell sorting was confirmed using beads and cells. Live single cells were sorted into 96 -well plates filled with $4 \mu \mathrm{L}$ of lysis buffer $(0.4 \%$ Triton, $2 \mathrm{U} / \mu \mathrm{L}$ RNase inhibitor (Clonotech, Takara), $7.5 \mu \mathrm{M}$ oligo dT (Biomers) and $10 \mathrm{mM}$ dNTP (ThermoFisher) [supplemented with 1:10,000,000 dilution of ERCC spike-in (ThermoFisher)]. Sorted cells were immediately vortexed, spun down and frozen on dry ice before storage at $-80^{\circ} \mathrm{C}$. Bulk populations were sorted into Eppendorfs containing $10 \mu \mathrm{L}$ of PBS, spun down, frozen on dry ice and stored at $-80^{\circ} \mathrm{C}$.

Quantification of intracellular bacteria. To quantify the replication of intracellular bacteria, infected host cells were washed with PBS, re-suspended in FACS buffer, stained and FACS sorted as described before. At each time point, 10 single cells per each experimental condition were sorted in 8-well strips containing $1 \%$ saponin (Sigma) in PBS and plated onto agar plates to determine the number of intracellular $\mathrm{CFU}$.

Confocal microscopy. MoDCs were plated in 8-well $\mu$-slides (Ibidi) coated with $0.01 \%$ poly-L-Lysine (Sigma) at a density of $2 \mathrm{E}+05$ cells per well in $250 \mu \mathrm{L}$ of RPMI supplemented with $10 \% \mathrm{FBS}$ and $2 \mathrm{mM}$ L-glutamine. Cells were infected with STM-LT2 or STM-D23580 and incubated for $2 \mathrm{~h}$ prior to fixation as described above.

Cells were washed twice with PBS, fixed with $4 \%$ paraformaldehyde (PFA; EMS) for $15 \mathrm{~min}$ and permeabilised with $0.05 \%$ Triton-X100 in PBS (Sigma) twice for $1 \mathrm{~min}$. Cells were blocked overnight at $4{ }^{\circ} \mathrm{C}$ with $250 \mu \mathrm{L}$ blocking solution $(5 \%$ human serum (Sigma), 5\% FBS) per well.

The following day, MoDCs were incubated with anti-Salmonella CSA-1 FITCconjugated antibody (KPL, Goat polyclonal, 1:100) in $140 \mu \mathrm{L}$ of blocking solution for $1 \mathrm{~h}$ at RT. Following two washes with PBS for $1 \mathrm{~min}$ each, cells were incubated with $200 \mu \mathrm{L}$ PBS containing $1 \mu \mathrm{g} / \mathrm{mL}$ DAPI (Thermo Fisher) for $10 \mathrm{~min}$ at RT. Finally, $10 \mu \mathrm{L}$ of VECTASHIELD Antifade Mounting Medium (H-100, Vector laboratories) were added to each well.

Images were acquired on a Zeiss LSM 880 inverted confocal microscope with a Plan-Apochromat 63x/1.40 Oil objective and processed using the Zeiss Zen software and assembled in Adobe illustrator.

Single-cell and bulk RNA-sequencing. Single-cell RNA-seq libraries were prepared as per the Smart-seq2 protocol by Picelli et al. ${ }^{17}$ with minor technical adaptations. Reverse transcription was carried on with $0.25 \mathrm{U} /$ reaction of SuperScript II (Invitrogen) and PCR pre-amplification with $5^{\prime}$ biotinylated IS PCR primers (Biomers) for 22 cycles. PCR cleaning was performed by using Ampure XP Beads (Beckman Coulter) at a ratio 0.8:1 (beads:cDNA). cDNA was re-suspended in elution buffer (Qiagen) and assessed with a High-sensitivity DNA chip (Agilent).

For Bulk RNA-seq RNA was extracted from 5000 sorted cells by using the Qiagen RNeasy Micro Kit according to the manufacturer's instructions. One microliter of RNA was added to the respective wells in the plate containing lysis buffer and processed as for single cells with the exception of the PCR preamplification step that was carried out for 18 cycles. Barcoded Illumina sequencing libraries (Nextera XT, Library preparation kit, Illumina) were generated using the automated platform (Biomek FXp). Finally, libraries were pooled, and sequencing was performed in paired-end mode for $2 \times 75$ bp cycles using the Illumina HiSeq 4000 sequencer.

RNA-sequencing read mapping and expression quantification. RNA-seq data were deposited in Gene Expression Omnibus under SuperSeries accession number GSE111546. Following deconvolution of RNA-seq raw reads into individual cells, initial quality assessment was performed using Fast $Q C$ (v0.11.4). Reads pairs were mapped to a composite genome made by concatenating the human genome reference sequence (GRCh38) and 92 ERCC ExFold RNA Spike-In Mixes sequences (ThermoFisher). Alignment was performed using HISAT2 (v2.0.3b) in default settings. Uniquely aligned read pairs were assigned and counted towards annotated human genomic genes and ERCC RNA spike-in features using featureCounts (v1.5.0-p2). All metrics were subsequently collected using MultiQC (v0.8) for reporting.

Quality control of single-cell RNA-sequencing data. Thirty-one cells ( $\sim 8 \%)$ were identified as outliers and removed from all downstream analyses. Briefly, outliers were detected for each quality metric (Supplementary Figure 6) beyond an appropriate number of median absolute deviations (MADs) above, below or further than the median value of all single cells, according to the nature of the metric: GC content ( $\pm 3 \mathrm{MADs})$, alignment rate ( $-3 \mathrm{MADs})$, library size ( \pm 3 MADs), percentage reads assigned to genes ( -3 MADs), library complexity for the top 50,100 and 200 genes, respectively ( $+3 \mathrm{MADs})$, count of detected genes ( $-2 \mathrm{MADs})$, ERCC content ( +5 MADs), mitochondrial gene content ( +3 MADs). Upon examination of endogenous genes, we observed similar distribution of average $\log _{10}$-transformed read count per million (CPM) values across all fifteen experimental conditions (time point, infection, status). On average, 10,820 genes (range: 9698-12,143) were detected above an average 1 CPM, and 4221 genes (range: 3636-4827) were detected below an average 1 CPM, respectively (Supplementary Figure $7 \mathrm{~A}$ ).

In addition, we observed good correlation between the average $\log _{2}$ transformed counts in cells from each condition and matched bulk samples prepared from 5000 cells sorted from MoDCs mock-infected or challenged with STM-LT2, STM-D23580 (Supplementary Figure 7B). Notably, although detected at very low abundance levels, ERCC spike-in features were more readily detected at higher read count levels in single cells relative to bulks, as expected from the higher relative proportion of ERCC spike-in molecules to endogenous RNA. We further confirmed a good correlation between the average counts of fragments yielded for each ERCC spike-in feature against the estimated count of molecules injected in each sample (Supplementary Figure 7C). ERCC spike-in molecules with an estimated count of injected molecules lower than one displayed an expected markedly larger variability and stochasticity of detection in individual cells. Overall, 8206 appreciably expressed endogenous genes were retained for downstream analyses ( $>10$ CPM in $\geq 25 \%$ of cells of $\geq 1$ experimental condition (Supplementary Figure 7D)).

Normalization of single-cell RNA-sequencing data. To account for the multifactorial nature of the experimental design and the considerable transcriptional changes expected over time, particularly in response to bacterial challenge, we estimated size factors for normalization from pools of cells sampled within unsupervised clusters. Specifically, four unsupervised clusters were identified by rank correlations between gene expression profiles using the quickCluster function of the scran package (v1.7.12). Notably, those unsupervised clusters largely recapitulated the three time points of the experimental design as well as the marked distinction between mock-infected and Salmonella-challenged MoDCs.

Dimensionality reduction and pseudotemporal ordering. Diffusion maps were computed using the runDiffusionMap wrapper function implemented in the scater package (v1.8.4), effectively computing a diffusion map from the 500 most variable genes in the data set using the destiny package (v2.10.2).

Pseudotemporal ordering was performed using the monocle package (v2.11.3). To isolate a set of ordering genes that define the progression of MoDCs through the infection challenge, we compared Salmonella-challenged cells collected at 2 and $6 \mathrm{~h}$ p.i. using the differentialGeneTest function. Next, we reduced data dimensionality using the DDRTree method and ordered cells along the resulting trajectory. Finally, we identified genes that change as a function of the computed pseudotime using the differentialGeneTest function. A $q$-value threshold of 0.001 was applied to all differential expression tests in the monocle workflow. Gene expression heat maps were produced using the ComplexHeatmap package (v1.18.1).

Identification of single-cell clusters. To identify clusters of cells in transcriptionally distinct states while controlling for the pronounced dynamics of gene expression over time, unsupervised clusters were computed separately at each time point by rank correlation between gene expression profiles using the quickCluster function of the scran package.

Single-cell differential expression analysis. The $s c d e$ package (v2.7.0) was used to estimate differential expression between groups of cells. Error models were 
computed separately for cells at each time point, while determining cross-fits and expected expression magnitudes separately within each experimental condition. The prior distribution for gene expression magnitudes was estimated separately for each time point using the scde.expression.prior function in default settings. $Z$ scores returned by $s c d e$ were converted to empirical $P$-values using the pnorm function of the R stats package (v3.5.0), and a significance cut-off $P<0.01$ was applied to define differentially expressed genes.

Quality control of small bulk RNA-sequencing data. Outliers were detected for each quality metric as described above for single-cell RNA-sequencing libraries. Multiple RNA-seq libraries from donor 3 appeared as outliers in multiple quality control metrics (Supplementary Figure 16). Consequently, all libraries generated from this donor were excluded to ensure a balanced experimental design for downstream differential expression analyses.

Overall, 8867 genes expressed on average above 10 CPM in at least one experimental condition (across the remaining two donors) were retained for downstream analysis.

Small bulk differential expression analysis. The DESeq2 package (v1.20.0) was used to estimate differential expression between experimental groups using the Negative Binomial GLM fitting and Wald statistics ${ }^{70}$. Size factors were estimated using the median ratio method, and dispersion estimates for the Negative Binomial distributed data were computed using a parametric dispersion-mean fit.

Quantitative PCR. MoDCs were stimulated with either STM-LT2 or STM-D23580 or left unstimulated. At 4 and $6 \mathrm{~h}$ p.i. single cells were FACS sorted in lysis buffer and CDNA was prepared according to Smart-seq2 protocol as described above. cDNA from eight individual cells from each experimental group was pooled and qPCR for selected genes was performed. qPCR reaction was carried on in 96-wellplate in $20 \mu \mathrm{L}$ final volume containing: Dual Lock DNA pol Master Mix (Life Technologies), forward and reverse primers (Supplementary Table 4) at a final concentration of $1 \mu \mathrm{M}$ each, $\mathrm{H}_{2} \mathrm{O}$ and $10 \mathrm{ng}$ of cDNA. After the initial denaturation steps at $50^{\circ} \mathrm{C}$ for $2 \mathrm{~min}$ and $95^{\circ} \mathrm{C}$ for $2 \mathrm{~min}$, PCR was performed for 40 cycles $\left(95^{\circ} \mathrm{C}\right.$ for $3 \mathrm{~s}$ and $60^{\circ} \mathrm{C}$ for $30 \mathrm{~s}$ for each cycle) by using the Quanti7 Studio machine. The specificity of primers was confirmed using Primer- BLAST (NCBI). Fold changes were determined using the $2^{-\Delta \mathrm{Ct}}$ method. The mRNA levels were expressed in relative copy numbers normalized against the Glyceraldehyde 3phosphate dehydrogenase (GAPDH) mRNA.

ELISAs. IL-1 $\beta$, IL-12p40 and IL-10 levels were measured from culture supernatants by ELISA kits (R\&D System) according to manufacturer's instructions. The range for IL- $1 \beta$ was $250-3.91 \mathrm{pg} / \mathrm{mL}$, for IL-12p40 was $4000-61.5 \mathrm{pg} / \mathrm{mL}$, and for IL-10 was $2000-31.3 \mathrm{pg} / \mathrm{mL}$. The results were expressed as $\mathrm{pg} / \mathrm{mL}$ for each cytokine.

Fluorescence-activated cell sorting (FACS) analysis. MoDCs were stimulated with either STM-LT2 or STM-D23580 or left unstimulated. At specific time points post infection, MoDCs were washed and incubated for $30 \mathrm{~min}$ with the following antibodies: anti-CD83 (PE; HB15e), anti-CD86 (PeCy7; IT2.2), anti-HLA-DR (APC; L243, or PerCP ef710; L243), HLA-A,B,C (APC Fire ${ }^{T M} 750$, w6/32) and anti-CD1a (FITC, HI149), all obtained from BioLegend. Zombie Aqua ${ }^{\mathrm{TM}}$ fixable viability dye (BioLegend) was used for exclusion of dead cells. After incubation, cells were washed in FACS buffer and fixed in 2\% PFA. Samples were acquired on a Fortessa X20 flow cytometer (BD Biosciences) and files were analysed on Flowjo (v.10.4.1).

T cell clone generation. Salmonella-specific $\mathrm{CD} 4^{+} \mathrm{T}$ cell clones were generated and expanded as previously described ${ }^{47}$. Briefly, CCR $7^{-} \mathrm{CD} 38^{+} \mathrm{CD} 4^{+} \mathrm{T}$ cells, isolated from volunteers after diagnosis of enteric fever, were sorted as single cells, expanded in vitro with phytohemagglutinin (PHA; Remel) and IL-2 in the presence of irradiated feeders for at least 3 weeks. Expanded clones were tested for their capacity of recognizing autologous Epstein Barr Virus (EBV)-transformed lymphoblastoid cell lines (EBV-LCL), either infected with live Salmonella or pulsed with Salmonella-related peptide pools (PhoN or HlyE). T cell clones were considered positive with IFN- $\gamma>5 \%$ in the presence of bacteria infected autologous cells, and/or IFN- $\gamma>20 \%$ in the presence of cells pulsed with proteins or peptide pools.

HLA-DRB1-restricted PhoN-specific $\mathrm{CD} 4^{+} \mathrm{T}$ cell clones cross-reactive against $S$. Typhimurium and $S$. Typhi serovars, as well as HlyE-specific $\mathrm{CD} 4^{+} \mathrm{T}$ cell clones reactive against $S$. Typhi, were used in this study.

Antigen presentation assay and intracellular staining. Human MoDCs were generated as described above, from $\mathrm{CD} 14^{+}$peripheral monocytes obtained from healthy blood donors, and screened for matching HLA-DRB1 haplotypes with the selected $\mathrm{T}$ cell clones. At $6 \mathrm{~h}$ p.i., MoDCs were stained for $30 \mathrm{~min}$ with anti-CD83 (PE; HB15e) and Zombie Aqua ${ }^{\mathrm{TM}}$ fixable viability dye to exclude dead cells. Cells were washed, re-suspended in FACS buffer and immediately sorted on a FACSAria III instrument. Infected cells were sorted based on their CD83 surface expression: namely, STM-D23580-infected MoDCs were sorted as CD83-llow or CD83 $3^{\text {high }}$, while STM-LT2 and ST-Ty2 infected MoDCs were sorted as CD83 ${ }^{+}$. Uninfected MoDCs were used as negative control.

Sorted live infected MoDCs were washed and co-cultured with Salmonellaspecific $\mathrm{CD}^{+} \mathrm{T}$ cell clones, at a ratio of 1:6 (MoDCs: $\mathrm{T}$ cells) in U-bottom 96-well plates (Costar), in RPMI supplemented with $10 \%$ FBS, $2 \mathrm{mM}$ L-Glut, $10 \mathrm{mM}$ HEPES (Life Technologies), 1 mM Sodium Pyruvate (Sigma), $0.01 \mathrm{mM}$ MEM Non Essential Amino acids (Life Technologies) and $50 \mu \mathrm{g} / \mathrm{mL}$ of gentamycin (MP Biomedicals). To enhance intracellular cytokine signals, $5 \mathrm{ng} / \mu \mathrm{L}$ of Brefeldin A (BioLegend) were added at $2 \mathrm{~h}$ after the beginning of co-culture. Following $14 \mathrm{~h}$ incubation, $\mathrm{T}$ cells were fixed, permeabilized and stained with the following antibodies: anti-CD3 (Alexa 700, UCHT1; BioLegend), anti-CD4 (APC ef780 RPA-T4; eBioscience), anti-CD40L (FITC, 24-31; BioLegend); anti TNF- $\alpha$ (PECy7, Mab11; BioLegend), anti- IFN- $\gamma$ (PE Dazzle, 4 S.B3; BioLegend). Viability was assessed with live/dead Aqua staining. Cells were acquired on a Fortessa X20 flow cytometer and data were analysed on FlowJo (v.10.4.1).

Statistical analysis. Statistical analyses were performed using GraphPad-Prism7 (GraphPad Software-San Diego, CA, United States). Pairwise comparisons were calculated using paired two-tailed Student's $t$-test. Differences among groups were determined by one-way or two-way ANOVA, as appropriate. ANOVA test statistics were corrected post-hoc by Tukey, applying a $95 \%$ confidence interval. A $P$-value $<0.05$ was considered statistically significant. All other analyses were performed in the $R$ statistical programming environment, using the latest version of $R$ and Bioconductor packages as appropriate.

\section{Data availability}

Sequences data have been deposited in the Gene Expression Omnibus under SuperSeries code GSE111546. The computational workflow has been made available at https://doi.org/10.5281/zenodo.1217158. All experimental data are available from the authors. A reporting summary for this Article is available as a Supplementary Information file.

Received: 25 April 2018 Accepted: 25 October 2018

Published online: 19 November 2018

\section{References}

1. Feasey, N. A., Dougan, G., Kingsley, R. A., Heyderman, R. S. \& Gordon, M. A Invasive non-typhoidal salmonella disease: an emerging and neglected tropical disease in Africa. Lancet 379, 2489-2499 (2012).

2. Majowicz, S. E. et al. The global burden of nontyphoidal Salmonella gastroenteritis. Clin. Infect. Dis. 50, 882-889 (2010).

3. Hendriksen, R. S. et al. Global monitoring of Salmonella serovar distribution from the World Health Organization Global Foodborne Infections Network Country Data Bank: results of quality assured laboratories from 2001 to 2007. Foodborne Pathog. Dis. 8, 887-900 (2011)

4. Kingsley, R. A. et al. Epidemic multiple drug resistant Salmonella Typhimurium causing invasive disease in sub-Saharan Africa have a distinct genotype. Genome Res 19, 2279-2287 (2009).

5. Leekitcharoenphon, P. et al. Genomics of an emerging clone of Salmonella serovar Typhimurium ST313 from Nigeria and the Democratic Republic of Congo. J. Infect. Dev. Ctries 7, 696-706 (2013).

6. Banchereau, J. et al. Immunobiology of dendritic cells. Annu Rev. Immunol. 18, 767-811 (2000)

7. Hammer, G. E. \& Ma, A. Molecular control of steady-state dendritic cell maturation and immune homeostasis. Annu Rev. Immunol. 31, 743-791 (2013).

8. Bueno, S. M., Gonzalez, P. A., Schwebach, J. R. \& Kalergis, A. M. T cell immunity evasion by virulent Salmonella enterica. Immunol. Lett. 111, 14-20 (2007).

9. Kiama, S. G. et al. Host cell responses of Salmonella typhimurium infected human dendritic cells. Immunol. Cell Biol. 84, 475-481 (2006).

10. Lapaque, N. et al. Salmonella regulates polyubiquitination and surface expression of MHC class II antigens. Proc. Natl Acad. Sci. USA 106, 14052-14057 (2009)

11. Jenner, R. G. \& Young, R. A. Insights into host responses against pathogens from transcriptional profiling. Nat. Rev. Microbiol. 3, 281-294 (2005).

12. Papalexi, E. \& Satija, R. Single-cell RNA sequencing to explore immune cell heterogeneity. Nat. Rev. Immunol. 18, 35-45 (2018).

13. Saliba, A. E., Westermann, A. J., Gorski, S. A. \& Vogel, J. Single-cell RNA-seq: advances and future challenges. Nucleic Acids Res 42, 8845-8860 (2014).

14. Kalisky, T., Blainey, P. \& Quake, S. R. Genomic analysis at the single-cell level. Annu Rev. Genet 45, 431-445 (2011).

15. Shalek, A. K. et al. Single-cell transcriptomics reveals bimodality in expression and splicing in immune cells. Nature 498, 236-240 (2013). 
16. Saliba, A. E. et al. Single-cell RNA-seq ties macrophage polarization to growth rate of intracellular Salmonella. Nat. Microbiol 2, 16206 (2016).

17. Picelli, S. et al. Full-length RNA-seq from single cells using Smart-seq2. Nat. Protoc. 9, 171-181 (2014).

18. Angerer, P. et al. Destiny: diffusion maps for large-scale single-cell data in R. Bioinformatics 32, 1241-1243 (2016).

19. Eisenreich, W., Heesemann, J., Rudel, T. \& Goebel, W. Metabolic host responses to infection by intracellular bacterial pathogens. Front Cell Infect. Microbiol 3, 24 (2013).

20. Lemaitre, B. \& Girardin, S. E. Translation inhibition and metabolic stress pathways in the host response to bacterial pathogens. Nat. Rev. Microbiol 11, 365-369 (2013).

21. Mohr, I. \& Sonenberg, N. Host translation at the nexus of infection and immunity. Cell Host Microbe 12, 470-483 (2012).

22. Lun, A. T., McCarthy, D. J. \& Marioni, J. C. A step-by-step workflow for lowlevel analysis of single-cell RNA-seq data with Bioconductor. F1000Res 5, 2122 (2016).

23. Shalek, A. K. et al. Single-cell RNA-seq reveals dynamic paracrine control of cellular variation. Nature 510, 363-369 (2014).

24. Kharchenko, P. V., Silberstein, L. \& Scadden, D. T. Bayesian approach to single-cell differential expression analysis. Nat. Methods 11, 740-742 (2014).

25. Bayer-Santos, E. et al. The Salmonella effector SteD mediates MARCH8dependent ubiquitination of MHC II molecules and inhibits T cell activation. Cell Host Microbe 20, 584-595 (2016).

26. Spano, S., Liu, X. \& Galan, J. E. Proteolytic targeting of Rab29 by an effector protein distinguishes the intracellular compartments of human-adapted and broad-host Salmonella. Proc. Natl Acad. Sci. USA 108, 18418-18423 (2011).

27. Gilchrist, M. et al. Systems biology approaches identify ATF3 as a negative regulator of Toll-like receptor 4. Nature 441, 173-178 (2006).

28. Whitmore, M. M. et al. Negative regulation of TLR-signaling pathways by activating transcription factor-3. J. Immunol. 179, 3622-3630 (2007).

29. Oh, J. et al. MARCH1-mediated MHCII ubiquitination promotes dendritic cell selection of natural regulatory T cells. J. Exp. Med 210, 1069-1077 (2013).

30. Stagg, H. R. et al. The TRC8 E3 ligase ubiquitinates MHC class I molecules before dislocation from the ER. J. Cell Biol. 186, 685-692 (2009).

31. Vilarino-Guell, C. et al. DNAJC13 mutations in Parkinson disease. Hum. Mol. Genet 23, 1794-1801 (2014).

32. Costa, L. F. et al. Iron acquisition pathways and colonization of the inflamed intestine by Salmonella enterica serovar Typhimurium. Int J. Med Microbiol 306, 604-610 (2016).

33. Kortman, G. A., Boleij, A., Swinkels, D. W. \& Tjalsma, H. Iron availability increases the pathogenic potential of Salmonella typhimurium and other enteric pathogens at the intestinal epithelial interface. PLoS ONE 7, e29968 (2012).

34. Biedzka-Sarek, M. \& El Skurnik, M. How to outwit the enemy: dendritic cells face Salmonella. APMIS 114, 589-600 (2006).

35. Bueno, S. M., Riquelme, S., Riedel, C. A. \& Kalergis, A. M. Mechanisms used by virulent Salmonella to impair dendritic cell function and evade adaptive immunity. Immunology 137, 28-36 (2012).

36. Ryan, E. J. et al. Dendritic cell-associated lectin-1: a novel dendritic cellassociated, C-type lectin-like molecule enhances T cell secretion of IL-4. J. Immunol. 169, 5638-5648 (2002).

37. Brown, G. D. Dectin-1: a signalling non-TLR pattern-recognition receptor. Nat. Rev. Immunol. 6, 33-43 (2006).

38. Bartee, E., Mansouri, M., Hovey Nerenberg, B. T., Gouveia, K. \& Fruh, K. Downregulation of major histocompatibility complex class I by human ubiquitin ligases related to viral immune evasion proteins. J. Virol. $\mathbf{7 8}$, $1109-1120$ (2004).

39. Goto, E. et al. c-MIR, a human E3 ubiquitin ligase, is a functional homolog of herpesvirus proteins MIR1 and MIR2 and has similar activity. J. Biol. Chem. 278, 14657-14668 (2003).

40. Tze, L. E. et al. CD83 increases MHC II and CD86 on dendritic cells by opposing IL-10-driven MARCH1-mediated ubiquitination and degradation. J. Exp. Med 208, 149-165 (2011).

41. Galbas, T., Steimle, V., Lapointe, R., Ishido, S. \& Thibodeau, J. MARCH1 down-regulation in IL-10-activated B cells increases MHC class II expression. Cytokine 59, 27-30 (2012).

42. Thibodeau, J. et al. Interleukin-10-induced MARCH1 mediates intracellular sequestration of MHC class II in monocytes. Eur. J. Immunol. 38, 1225-1230 (2008).

43. De Angelis Rigotti, F. et al. MARCH9-mediated ubiquitination regulates MHC I export from the TGN. Immunol. Cell Biol. 95, 753-764 (2017).

44. Cao, X. et al. CD1 molecules efficiently present antigen in immature dendritic cells and traffic independently of MHC class II during dendritic cell maturation. J. Immunol. 169, 4770-4777 (2002).

45. Zajonc, D. M. et al. Molecular mechanism of lipopeptide presentation by CD1a. Immunity 22, 209-219 (2005).
46. Prechtel, A. T., Turza, N. M., Theodoridis, A. A. \& Steinkasserer, A. CD83 knockdown in monocyte-derived dendritic cells by small interfering RNA leads to a diminished T cell stimulation. J. Immunol. 178, 5454-5464 (2007).

47. Napolitani, G. et al. Clonal analysis of Salmonella-specific effector T cells reveals serovar-specific and cross-reactive T cell responses. Nat. Immunol. 19, 742-754 (2018).

48. Oscarsson, J. et al. Characterization of a pore-forming cytotoxin expressed by Salmonella enterica serovars typhi and paratyphi A. Infect. Immun. 70, 5759-5769 (2002)

49. Lokken, K. L. et al. Malaria parasite infection compromises control of concurrent systemic non-typhoidal Salmonella infection via IL-10-mediated alteration of myeloid cell function. PLoS Pathog. 10, e1004049 (2014).

50. Matsuki, Y. et al. Novel regulation of MHC class II function in B cells. $E M B O$ J. 26, 846-854 (2007).

51. Cheminay, C., Mohlenbrink, A. \& Hensel, M. Intracellular Salmonella inhibit antigen presentation by dendritic cells. J. Immunol. 174, 2892-2899 (2005).

52. Yu, Q. et al. Association of Neisseria gonorrhoeae Opa(CEA) with dendritic cells wresses their ability to elicit an HIV-1-specific T cell memory response. PLoS ONE 8, e56705 (2013).

53. Kummer, M. et al. Herpes simplex virus type 1 induces CD83 degradation in mature dendritic cells with immediate-early kinetics via the cellular proteasome. J. Virol. 81, 6326-6338 (2007).

54. Engelmayer, J. et al. Vaccinia virus inhibits the maturation of human dendritic cells: a novel mechanism of immune evasion. J. Immunol. 163 6762-6768 (1999).

55. Salio, M., Cella, M., Suter, M. \& Lanzavecchia, A. Inhibition of dendritic cell maturation by herpes simplex virus. Eur. J. Immunol. 29, 3245-3253 (1999).

56. Senechal, B., Boruchov, A. M., Reagan, J. L., Hart, D. N. \& Young, J. W. Infection of mature monocyte-derived dendritic cells with human cytomegalovirus inhibits stimulation of T-cell proliferation via the release of soluble CD83. Blood 103, 4207-4215 (2004).

57. Heilingloh, C. S. et al. The major immediate-early protein IE2 of human cytomegalovirus is sufficient to induce proteasomal degradation of CD83 on mature dendritic cells. Front. Microbiol. 8, 119 (2017).

58. Kruse, M. et al. Mature dendritic cells infected with herpes simplex virus type 1 exhibit inhibited T-cell stimulatory capacity. J. Virol. 74, 7127-7136 (2000).

59. Das, S. et al. Expression of B7-H1 on gastric epithelial cells: its potential role in regulating $\mathrm{T}$ cells during Helicobacter pylori infection. J. Immunol. 176, 3000-3009 (2006).

60. Ross, P. J., Lavelle, E. C., Mills, K. H. \& Boyd, A. P. Adenylate cyclase toxin from Bordetella pertussis synergizes with lipopolysaccharide to promote innate interleukin-10 production and enhances the induction of Th2 and regulatory T cells. Infect. Immun. 72, 1568-1579 (2004).

61. Skinner, J. A., Reissinger, A., Shen, H. \& Yuk, M. H. Bordetella type III secretion and adenylate cyclase toxin synergize to drive dendritic cells into a semimature state. J. Immunol. 173, 1934-1940 (2004).

62. Marriott, I., Thomas, E. K. \& Bost, K. L. CD40-CD40 ligand interactions augment survival of normal mice, but not CD40 ligand knockout mice, challenged orally with Salmonella dublin. Infect. Immun. 67, 5253-5257 (1999).

63. Schreiber, H. A. et al. Dendritic cells in chronic mycobacterial granulomas restrict local anti-bacterial $\mathrm{T}$ cell response in a murine model. PLOS ONE 5, e11453 (2010)

64. Murray, R. A., Siddiqui, M. R., Mendillo, M., Krahenbuhl, J. \& Kaplan, G. Mycobacterium leprae inhibits dendritic cell activation and maturation. J. Immunol. 178, 338-344 (2007).

65. Yamauchi, P. S. et al. A role for CD40-CD40 ligand interactions in the generation of type 1 cytokine responses in human leprosy. J. Immunol. 165, $1506-1512$ (2000)

66. Avraham, R. et al. Pathogen cell-to-cell variability drives heterogeneity in host immune responses. Cell 162, 1309-1321 (2015).

67. Avital, G. et al. scDual-Seq: mapping the gene regulatory program of Salmonella infection by host and pathogen single-cell RNA-sequencing. Genome Biol. 18, 200 (2017).

68. Carden, S. E. et al. Pseudogenization of the secreted effector gene sseI confers rapid systemic dissemination of S. Typhimurium ST313 within migratory dendritic cells. Cell Host Microbe 21, 182-194 (2017).

69. McClelland, M. et al. Complete genome sequence of Salmonella enterica serovar Typhimurium LT2. Nature 413, 852-856 (2001).

70. Love, M. I., Huber, W. \& Anders, S. Moderated estimation of fold change and dispersion for RNA-seq data with DESeq2. Genome Biol. 15, 550 (2014).

\section{Acknowledgements}

A.A. and G.N., were funded by UK Medical Research Council (MRC). L.P.-L. was supported by the MRC and Oxford NIHR biomedical Research Centre. A.S. was supported by the MRC, an NIHR Research Professorship and Wellcome Trust Investigator 
Award. We would like to acknowledge Paul Sopp and Craig Waugh in the flow cytometry facility at the Weatherall Institute of Molecular Medicine. The facility is supported by the MRC HIU; MRC MHU (MC_UU_12009); NIHR Oxford BRC and John Fell Fund (131/030 and 101/517), the EPA fund (CF182 and CF170) and by the WIMM Strategic Alliance awards G0902418 and MC_UU_12025. We acknowledge support of the Oxford Single Cell Consortium and thank the Oxford Genomics Centre at the Wellcome Centre for Human Genetics (funded by Wellcome Trust grant reference 203141/Z/16/Z) for the generation and initial processing of the sequencing data. We thank Life Science Editors for editing assistance.

\section{Author contributions}

A.A. and L.P.-L. conceived, designed and performed experiments. K.R.A. performed computational analyses. A.C. and D.S. provided support for computational analyses. L. P.-L. performed flow cytometry experiments. N.A. assisted in single-cell sequencing experiments. G.N. assisted with experimental design and manuscript editing. J.K. and C. L. performed confocal microscopy experiments. T.A. provided reagents. A.A., K.R.A. and A.S. wrote the manuscript. M.G. provided advice. A.S. conceived the study, obtained funding and supervised the work.

\section{Additional information}

Supplementary Information accompanies this paper at https://doi.org/10.1038/s41467018-07329-0.
Competing interests: The authors declare no competing interests.

Reprints and permission information is available online at http://npg.nature.com/ reprintsandpermissions/

Publisher's note: Springer Nature remains neutral with regard to jurisdictional claims in published maps and institutional affiliations.

\section{(c) (i)}

Open Access This article is licensed under a Creative Commons Attribution 4.0 International License, which permits use, sharing, adaptation, distribution and reproduction in any medium or format, as long as you give appropriate credit to the original author(s) and the source, provide a link to the Creative Commons license, and indicate if changes were made. The images or other third party material in this article are included in the article's Creative Commons license, unless indicated otherwise in a credit line to the material. If material is not included in the article's Creative Commons license and your intended use is not permitted by statutory regulation or exceeds the permitted use, you will need to obtain permission directly from the copyright holder. To view a copy of this license, visit http://creativecommons.org/licenses/by/4.0/.

(C) The Author(s) 2018 